\title{
Muscle-derived interleukin 6 increases exercise capacity by signaling in osteoblasts
}

\author{
Subrata Chowdhury, ${ }^{1}$ Logan Schulz, ${ }^{1}$ Biagio Palmisano, ${ }^{1}$ Parminder Singh, ${ }^{2}$ Julian M. Berger, ${ }^{1}$ Vijay K. Yadav, ${ }^{1,2}$ Paula Mera, ${ }^{1,3,4,5}$ \\ Helga Ellingsgaard, ${ }^{6,7}$ Juan Hidalgo, ${ }^{8}$ Jens Brüning, ${ }^{9}$ and Gerard Karsenty ${ }^{1}$ \\ 'Department of Cenetics and Development, Vagelos College of Physicians and Surgeons, Columbia University, New York, New York, USA. ${ }^{2}$ National Institute of Immunology, New Delhi, India. ${ }^{3}$ Department \\ of Biochemistry and Physiology, School of Pharmacy and Food Sciences, ${ }^{4}$ Institut de Biomedicina de la Universitat de Barcelona (IBUB), Universitat de Barcelona, Barcelona, Spain. ${ }^{5}$ Centro de Investigación \\ Biomédica en Red de Fisiopatología de la Obesidad y la Nutrición (CIBEROBN), Instituto de Salud Carlos III, Madrid, Spain. ${ }^{6}$ Centre of Inflammation and Metabolism and ${ }^{7}$ Centre for Physical Activity \\ Research, Rigshospitalet, University of Copenhagen, Copenhagen, Denmark. ${ }^{8}$ Department of Cellular Biology, Physiology and Immunology, Faculty of Biosciences, Universitat Autònoma de Barcelona, \\ Barcelona, Spain. ${ }^{9}$ Max Planck Institute for Metabolism Research, Cologne, Germany.
}

\begin{abstract}
Given the numerous health benefits of exercise, understanding how exercise capacity is regulated is a question of paramount importance. Circulating interleukin 6 (IL-6) levels surge during exercise and IL-6 favors exercise capacity. However, neither the cellular origin of circulating IL- 6 during exercise nor the means by which this cytokine enhances exercise capacity has been formally established yet. Here we show through genetic means that the majority of circulating IL- 6 detectable during exercise originates from muscle and that to increase exercise capacity, IL-6 must signal in osteoblasts to favor osteoclast differentiation and the release of bioactive osteocalcin in the general circulation. This explains why mice lacking the IL-6 receptor only in osteoblasts exhibit a deficit in exercise capacity of similar severity to the one seen in mice lacking musclederived IL-6 (mIL-6), and why this deficit is correctable by osteocalcin but not by IL-6. Furthermore, in agreement with the notion that IL- 6 acts through osteocalcin, we demonstrate that mIL-6 promotes nutrient uptake and catabolism into myofibers during exercise in an osteocalcin-dependent manner. Finally, we show that the crosstalk between osteocalcin and IL-6 is conserved between rodents and humans. This study provides evidence that a muscle-bone-muscle endocrine axis is necessary to increase muscle function during exercise in rodents and humans.
\end{abstract}

\section{Introduction}

The ability to exercise, or exercise capacity, is an evolutionarily conserved physiological function of vital importance because it allows animals living in the wild to escape danger. In addition, exercise provides numerous health benefits to the general population. This explains why there is a growing interest in understanding how this physiological process is regulated $(1,2)$. Several cytokines have been shown to favor muscle function during exercise and as a result to increase exercise capacity (3). One of them, interleukin 6 (IL-6), sees its circulating levels surge during exercise (4-8). Because $I l 6$ is expressed by many cell types, this raises the question of the identity of the cell type(s) responsible for its increase in the general circulation during exercise. It has been proposed that once secreted into the general circulation, IL- 6 enhances exercise capacity by promoting gluconeogenesis and lipolysis, i.e., the production of the 2 main nutrients for myofibers (3, 7, 9-12). However, this mechanism of action has not been verified in vivo, which leaves open the possibility that IL-6 could regulate exercise capacity through other, yet to be described, means. Those 2 questions are of paramount importance if we want to achieve a comprehensive understanding of how exercise capacity is regulated.

Conflict of interest: The authors have declared that no conflict of interest exists. Copyright: (5) 2020, American Society for Clinical Investigation.

Submitted: September 17, 2019; Accepted: February 11, 2020; Published: April 27, 2020. Reference information: J Clin Invest. 2020;130(6):2888-2902.

https://doi.org/10.1172/JCI133572.
Hormones signaling in myofibers can also contribute to the increase in muscle function during exercise. In that regard, we have shown, through its injections in wild-type (WT) mice and the analysis of mice lacking its receptor only in myofibers, that the bone-derived hormone osteocalcin is necessary and sufficient to increase muscle function during exercise in the mouse (13). Osteocalcin also promotes the rise in $\mathrm{Il6}$ expression in muscle and in circulating IL-6 levels during exercise. On the other hand, in cell culture experiments, IL-6 increased the expression in osteoblasts of Rankl, a gene necessary for osteoclast differentiation, a process that is required for the generation of the uncarboxylated and bioactive form of osteocalcin (13-15).

Altogether, the functions of IL-6 during exercise and the relationship between osteocalcin and muscle-derived IL-6 (mIL-6) raise the following questions regarding the mechanism(s) whereby IL- 6 promotes exercise capacity: To what extent does muscle contribute to the rise in circulating IL- 6 during exercise? Does mIL-6 enhance exercise capacity on its own? If it does, what is its mechanism of action? Can we provide evidence that regulatory events identified in the mouse also take place in humans? The present study was conducted to address these questions.

\section{Results}

IL-6 is necessary for the increase in circulating osteocalcin levels observed in response to a training intervention in humans. If the interplay identified in the mouse and taking place during exercise between IL- 6 and osteocalcin is of real physiological significance 

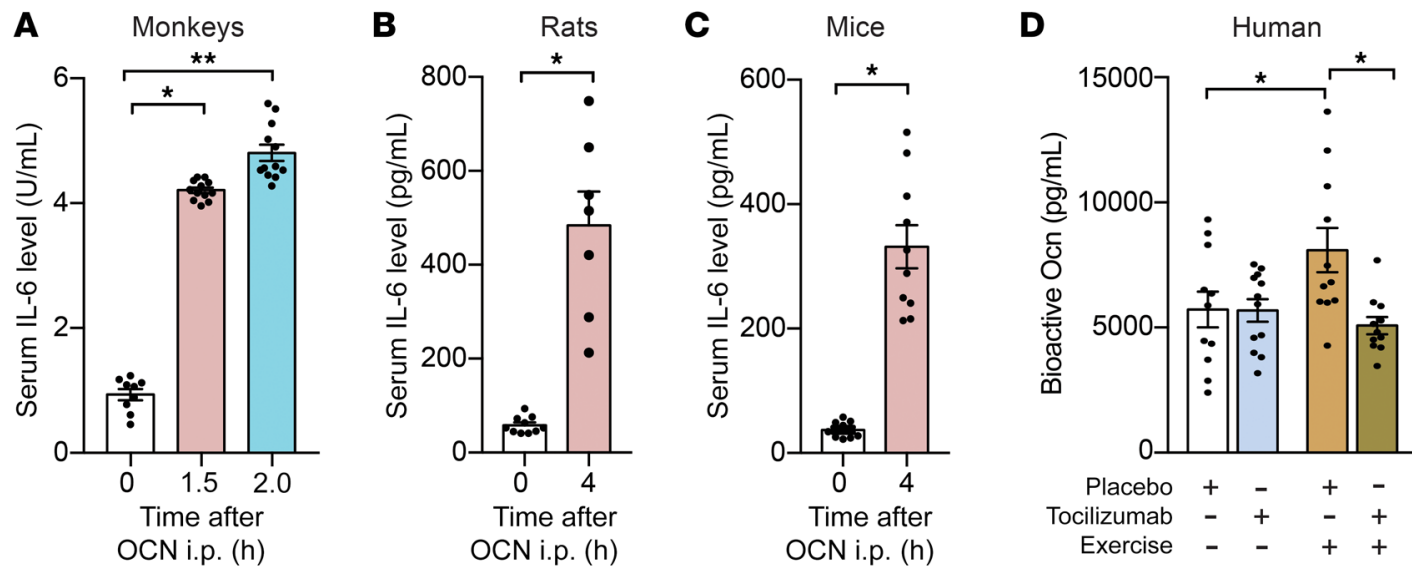

Figure 1. IL-6 is necessary for the increase in circulating osteocalcin levels observed in response to a training intervention in humans. (A-C) Circulating IL-6 levels in (A) rhesus monkeys ( $14 \pm 0.8$ years old), (B) rats (4 months old), and (C) mice (3 months old) treated with vehicle or osteocalcin (0cn) (13.5 $\mathrm{ng} / \mathrm{g}$ for monkeys, $30 \mathrm{ng} / \mathrm{g}$ for rats, and $30 \mathrm{ng} / \mathrm{g}$ for mice). $n=12$ per treatment. Statistical analyses were conducted using 1-way ANOVA followed by Tukey's post hoc test (A) or 2-tailed unpaired $t$ test (B and C). (D) Circulating uncarboxylated and bioactive Ocn levels in obese, nondiabetic subjects treated with either placebo or a neutralizing antibody against the IL-6 receptor in combination with or without intensive endurance exercise for 45 minutes, 3 times a week, for 12 consecutive weeks. $n=11$ per group except no exercise with the tocilizumab group, $n=12$. Statistical analyses were conducted using 1-way ANOVA followed by the Holm-Sidak post hoc test. All results presented as the mean $\pm \mathrm{SEM} .{ }^{*} P<0.05 ;{ }^{* *} P<0.01$.

in determining exercise capacity, it should be observed in other species, including primates (13). In a first test of this contention we found that, as it does in mice, a single injection of recombinant osteocalcin significantly increased circulating IL-6 levels in rats and more importantly from a clinical vantage point, in nonhuman primates (Figure $1, \mathrm{~A}-\mathrm{C}$ ).

These results led us to further expand our analysis and to measure circulating levels of bioactive osteocalcin in obese, nondiabetic human subjects that had either rested or had undergone a 12-weeklong aerobic training period (8). This 12 -week training period consisted of endurance exercise sessions ( 45 minutes of biking) 3 times a week. We used for that purpose a novel and sensitive ELISA that specifically measures circulating uncarboxylated and bioactive human osteocalcin $(13,14)$. This endurance exercise training protocol resulted in a $60 \%$ increase in circulating osteocalcin levels in these individuals compared with resting individuals. Of note, this increase was sustained for at least 2 days after the cessation of exercise. To the best of our knowledge, this represents the first evidence that performing endurance exercise 3 times a week for a relatively long period of time ( 3 months) is enough to induce a significant increase in circulating osteocalcin levels in humans (Figure 1D).

In the third experiment, we measured circulating osteocalcin levels in cohorts of exercising obese nondiabetic subjects that received either placebo or an anti-IL-6 receptor (anti-IL-6R) antibody (tocilizumab) during the training period (8). We observed that in individuals that had received the anti-IL-6R antibody, the increase in circulating osteocalcin levels triggered by exercise had virtually disappeared (Figure 1D). Albeit of a correlative nature, these latter data are an indication that IL-6 may regulate circulating osteocalcin levels in humans as well during exercise.

mIL- 6 is needed for maximal exercise capacity. In view of the conservation of regulation between mice and humans presented above, we asked to what extent does muscle contribute to the increase in circulating IL-6 levels observed during exercise and does mIL- 6 actually regulate exercise capacity?
For that purpose we crossed Il $6^{f / f l}$ mice with Hsa-MerCreMer mice that express the Cre recombinase in an inducible manner specifically in myofibers (Figure 2A and refs. 16, 17). Cre expression was induced in 5-week-old mice by intraperitoneal (i.p.) injections of tamoxifen $(10 \mathrm{mg} / \mathrm{mL}) 4$ days in a row followed by feeding with tamoxifen-containing chow diet $(1 \mathrm{mg} / 20 \mathrm{~g}$ of body weight) for 2 to 4 weeks before analysis (18). This resulted in a highly efficient deletion of Il6 in oxidative (soleus), glycolytic (extensor digitalis or EDL), and mixed-fiber muscle (gastrocnemius). In contrast, no deletion of Il6 could be detected in the heart or any other tissues tested (Figure 2, B and C). $\mathrm{Il}_{\mathrm{Hsa}}{ }^{-1-}$ mice were obtained at the expected Mendelian ratio, had a normal life span, and appeared overtly normal.

The contribution of muscle to circulating IL-6 levels during exercise was assessed by measuring its levels in male and female $\mathrm{IlO}_{\mathrm{Ha}}{ }^{-1-}$ and control mice before and after exercise (running on a treadmill at a constant speed until exhaustion or for 50 minutes) (13). We observed that although circulating IL-6 levels increased robustly in control mice after exercise, they did not in $\mathrm{Il}_{\mathrm{Hsa}}{ }^{-1}$ mice (Figure 2D and Supplemental Figure 1, A and B). These results indicate that the vast majority of the IL- 6 molecules present in general circulation during exercise originate from muscle.

The extent to which mIL- 6 contributes to exercise capacity during an endurance exercise was determined by measuring the ability of $\mathrm{Il}_{\mathrm{Hsa}}{ }^{-1-}$ and control mice to perform endurance running. Starting at 3 months of age, male and female $\mathrm{Il}_{\mathrm{Hsa}}{ }^{-1-}$ mice exhibited a significant reduction in the time and distance they run on a treadmill apparatus when compared with control littermates (Figure 2E and Supplemental Figure 1C). The defect in exercise capacity observed in $\mathrm{Il}_{\mathrm{Hsa}}{ }^{-1-}$ mice was specific to endurance exercise because grip strength was not affected by the inactivation of mIL-6 (Supplemental Figure 1, D and E). As inferred by these results, treating WT mice with an antibody against IL-6 significantly decreased their exercise capacity (Figure $2 \mathrm{~F}$ ). The severity of the deficit in exercise capacity observed in $\mathrm{Il}_{\mathrm{Hsa}}^{-1-}$ mice was an incentive to explore its cellular and molecular bases. 
A

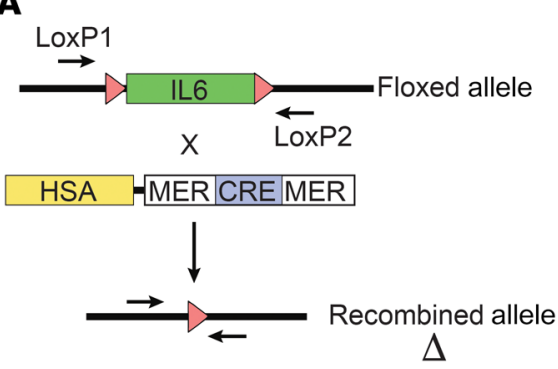

B

E

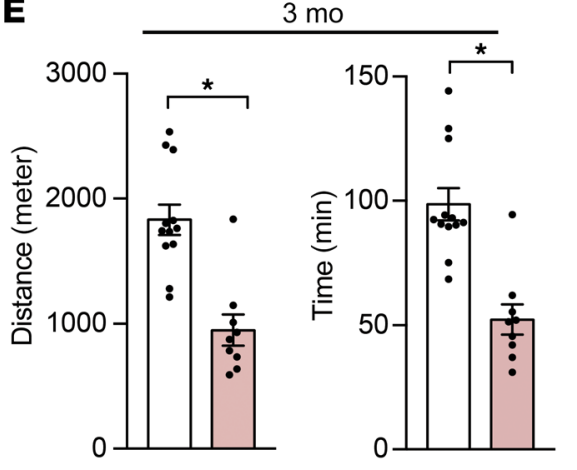

D
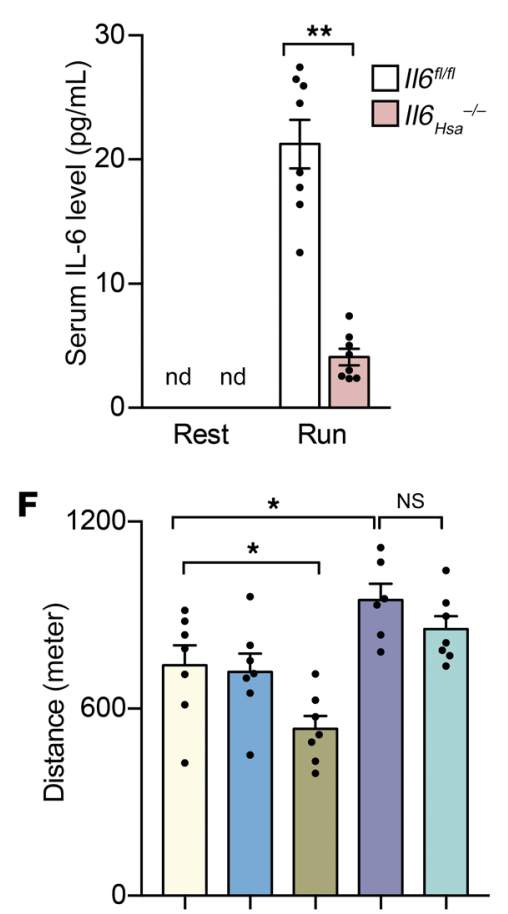

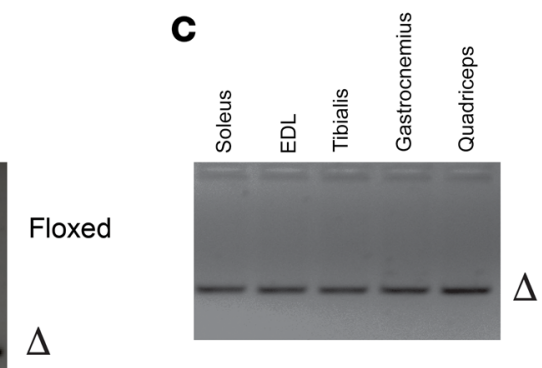

$\Delta$
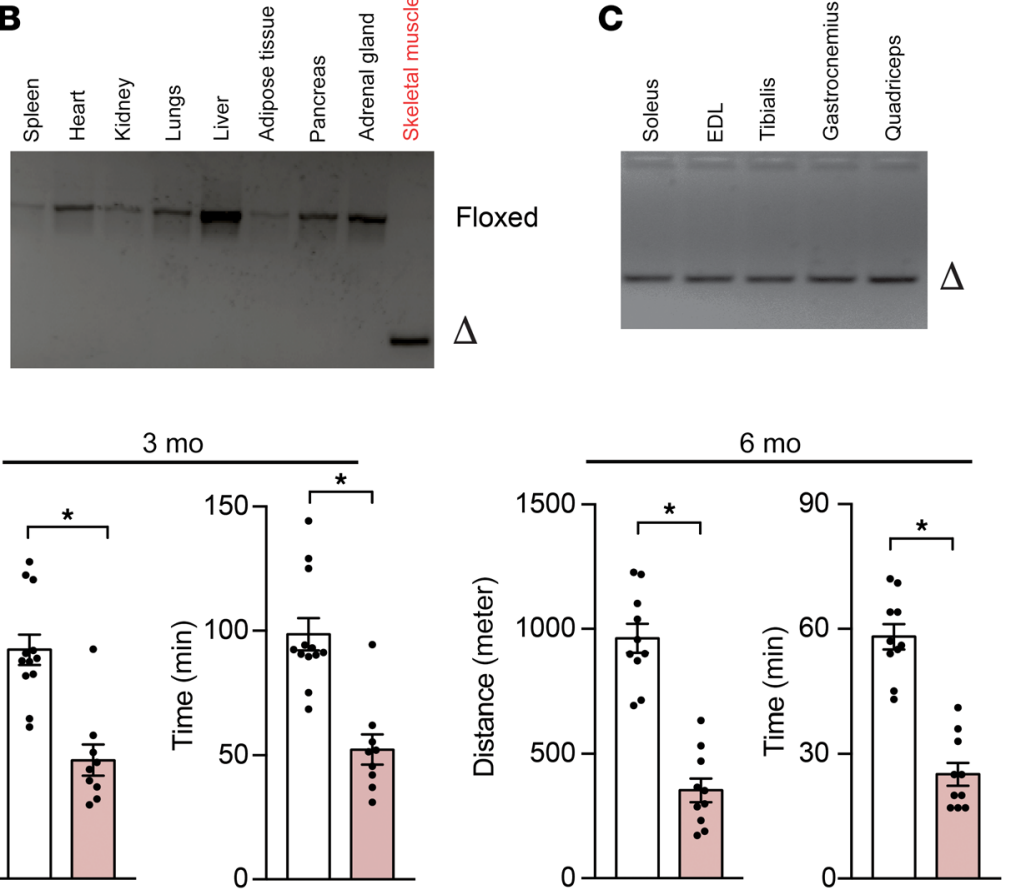

Figure 2. Muscle-derived IL-6 is needed for maximal exercise capacity. (A) The promoter of the human $\alpha$-skeletal actin (HSA) gene drives expression of the MerCreMer (MCM) gene, which harbors a mutated estrogen receptor (Mer) ligand-binding domain on each end of Cre recombinase. After crossing with $/ / 6^{f / f f l}$ mice and treatment with tamoxifen, a Cre-mediated recombination event results in the deletion of the $I / 6$ gene. (B) Detection of $I / 6$ deletion by PCR on genomic DNA isolated from various tissues of $1 / 6_{H s a}{ }^{-1-}$ mice. (C) Detection of $/ 16$ deletion by PCR on genomic DNA isolated from various skeletal muscles of $1 / 6_{H s a}{ }^{-1-}$ mice. (D) Circulating IL-6 levels in 3-month-old male $I / 6^{f / f f}$ and $I I 6_{H s a}^{-1-}$ mice at rest and after exercise. $n=8$ per group. (E) Performance during an endurance run of 3 - and 6-month-old $I / 6^{f / / f l}$ and $/ 16_{H s a}{ }^{-1-}$ female mice. (F) Performance during an endurance test (running on a treadmill at $30 \mathrm{~cm} / \mathrm{s}$ until exhaustion) of 8-month-old mice treated with vehicle or osteocalcin $(0 \mathrm{cn}, 500 \mathrm{ng} / \mathrm{g})$ and an antibody against IL-6 or a control IgG, $n=7$ per group. Results presented as the mean \pm SEM. Data were analyzed with 2-tailed unpaired $t$ test ( $\mathbf{D}$ and $\mathbf{E}$ ) or 1-way ANOVA followed by Tukey's post hoc test $(\mathbf{F}){ }^{*} P<0.05$; ${ }^{* *} P<0.01$.

mIL-6 favors exercise capacity in part through osteocalcin. Although trying to unravel how mIL-6 enhances exercise capacity, we observed that circulating osteocalcin (encoded by the Ocn gene) levels did not increase in $\mathrm{IlO}_{\mathrm{Hsa}}{ }^{-/-}$mice after exercise, as they did in control mice (Figure 3A). Moreover, $\mathrm{OCn}^{+/-} \mathrm{Il}_{\mathrm{Hsa}}{ }^{+-}$mice exhibited a deficit in exercise capacity as severe as the one observed in $\mathrm{Il}_{\mathrm{Hsa}}{ }^{-1-}$ mice, whereas single heterozygous mice did not, and that circulating osteocalcin did not increase in $\mathrm{Ocn}^{+/-} \mathrm{Il}_{\mathrm{Hsa}}{ }^{+/-}$mice during an endurance exercise. This genetic epistasis experiment indicated that osteocalcin mediates mIL- 6 regulation of adaptation to exercise in the mouse, as it appears to do in humans (Figure 3, B and C).

If the aforementioned hypothesis is accurate, then one would expect that osteocalcin would correct, at least in part, the deficit in exercise capacity observed in mice lacking mIL-6. In a positive control experiment, we observed that an injection of IL- 6 before exercise increased circulating IL-6 levels, corrected as it should the deficit in exercise capacity observed in the $\mathrm{IlO}_{\mathrm{Hsa}}{ }^{-1}$ mice, and restored the surge of circulating osteocalcin levels during endurance exercise (Figure 3, D-F). This IL-6 injection also corrected the deficit in exercise capacity seen in $\mathrm{Ocn}^{+/-} \mathrm{Il}_{\mathrm{Hsa}}^{+-}$mice (Figure 3G). More importantly for our purpose, an injection of osteocalcin corrected the majority of the deficit in exercise capacity seen in $\mathrm{IlO}_{\mathrm{Hsa}}{ }^{-1}$ mice during endurance exercise. Of note, circulating Il-6 levels increased following these osteocalcin injections, suggesting that osteocalcin can release IL-6 from organs other than muscle (Figure $3, \mathrm{H}$ and I). These data support the notion that osteocalcin is a mediator of mIL-6's ability to enhance exercise capacity without excluding the possibility that other mediators of this action exist. 
A
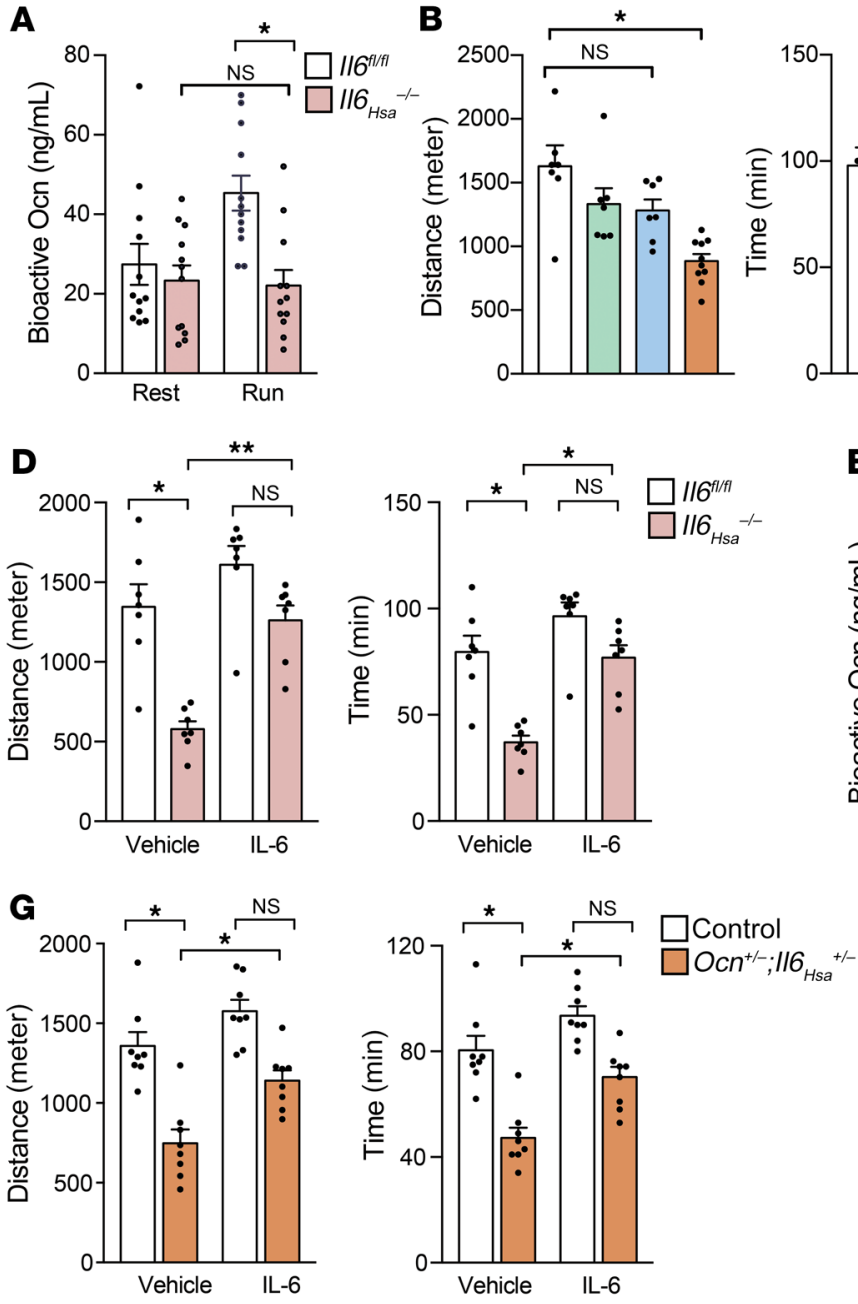

E
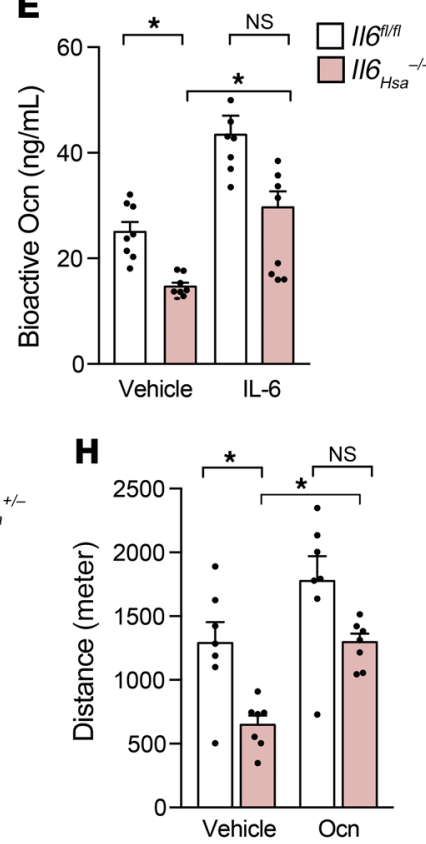

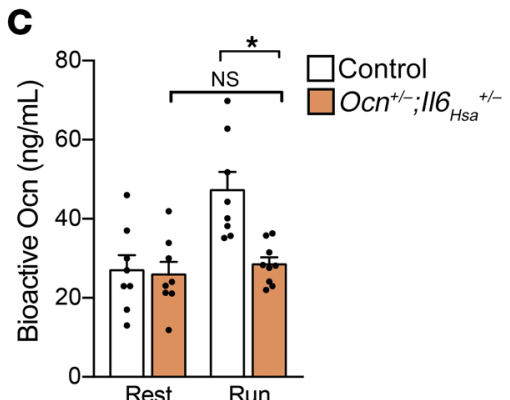

$\mathbf{F}$
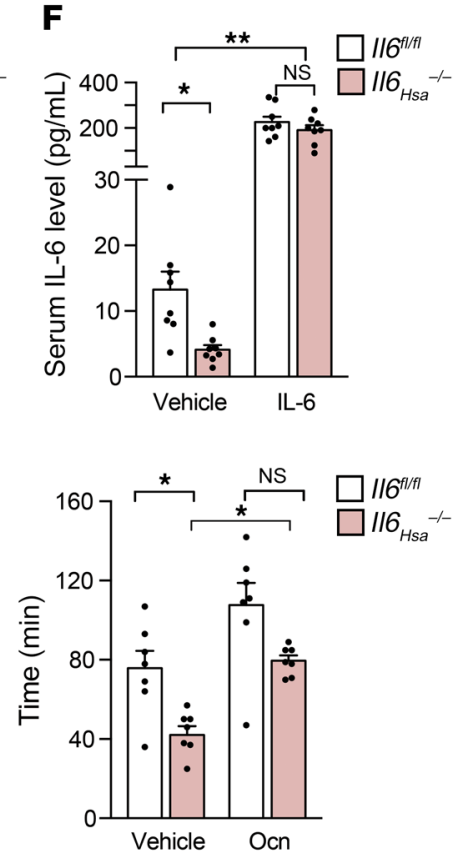

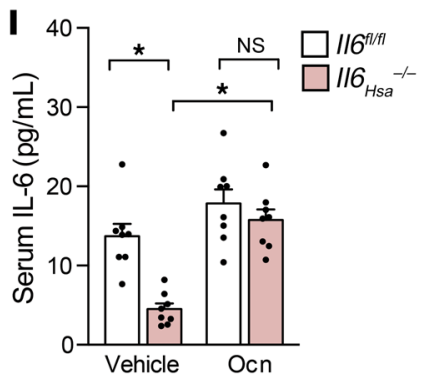

Figure 3. mIL-6 favors exercise capacity in part through osteocalcin. (A) Circulating bioactive osteocalcin (Ocn) levels in 3-month-old $/ / 6^{f l / f l}$ and $/ / 6_{H s a}{ }^{-/-}$

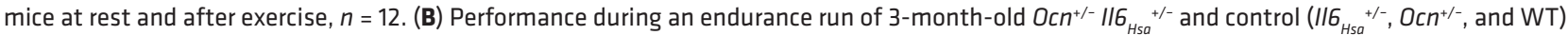
mice, $n=8-12$. (C) Circulating Ocn levels in 3-month-old control ( $/ / 6^{f l / f l}, 0 \mathrm{cn}{ }^{+/-}$, and WT mice) and $0 \mathrm{cn}{ }^{+/-} / 16_{H s a}{ }^{+/-}$mice at rest and after exercise, $n=8$. (D) Performance during an endurance run of 3-month-old $/ / 6^{f / f l}$ and $/ / \sigma_{H s a}^{-1-}$ mice after an i.p. injection of IL- 6 (3 ng/g), $n=8$. (E) Circulating Ocn levels during an endurance run in 3-month-old $I / 6^{f l / f l}$ and $I / 6_{H s a}{ }^{-/-}$mice after an i.p. injection of IL- 6 (3 ng/g), $n=8$. (F) Circulating IL-6 levels during an endurance run in 3-month-old $/ / 6^{f l / f l}$ and $/ / \sigma_{H s a}^{-/-}$mice after an i.p. injection of IL-6 (3 ng/g), $n=8$. (G) Performance during an endurance run of 3-month-old control ( $/ / \sigma^{f l / f l}$, $\mathrm{Ocn}^{+/-}$, and WT mice) and $\mathrm{Ocn}{ }^{+/-} / 1 \sigma_{\mathrm{Hsa}^{+/-}}$mice after an i.p. injection of IL-6 (3 ng/g), $n=8$. (H) Performance during an endurance run of 3-month-old $/ / 6^{f l / f l}$ and $/ / \sigma_{H s a}^{-1-}$ mice after an i.p. Ocn injection (120 ng/g), $n=7$. (I) Circulating IL-6 levels during an endurance run in 3-month-old $/ / 6^{f l / f l}$ and $/ / 6_{H s a}{ }^{-/-}$mice after an i.p. injection of $\mathrm{Ocn}(120 \mathrm{ng} / \mathrm{g}), n=8$. These results are representative of 3 independent experiments. Data were analyzed by 1 -way ANOVA followed by Tukey's post hoc test. Results presented as the mean \pm SEM. ${ }^{*} P<0.05 ;{ }^{*} P<0.01$.

mIL-6 contributes to the maintenance of muscle mass in an osteocalcin-independent manner. In the course of this analysis we observed a second muscle phenotype in $\mathrm{Il}_{\mathrm{HS}}{ }^{-1-}$ mice. Indeed, and even though myostatin (Mstn) expression was not affected (Supplemental Figure 1E), $\mathrm{IlO}_{\mathrm{Hs}}{ }^{-1}$ - mice exhibited a significant decrease in the weight of oxidative muscles, e.g., the soleus, the most mobilized muscle type during an endurance exercise, in 3-month-old $\mathrm{IlO}_{\mathrm{Hsa}}{ }^{-1-}$ mice compared with control littermates (Figure 4A). One experimental line of evidence indicates, however, that mIL-6 regulates muscle function independently of its regulation of muscle 
A

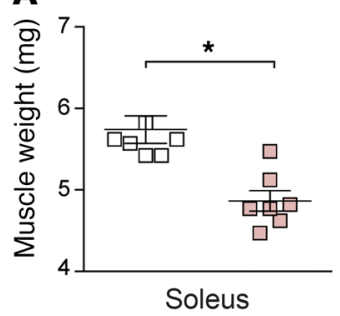

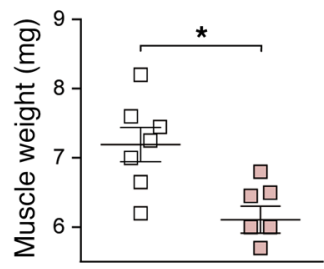

EDL

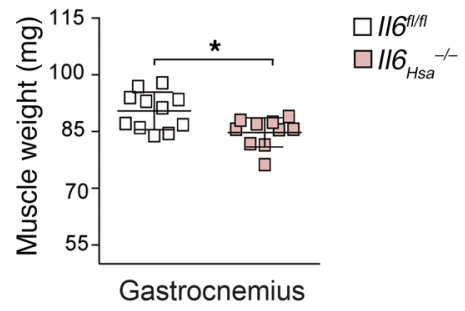

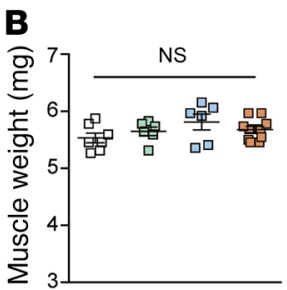

Soleus

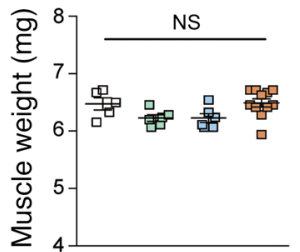

EDL

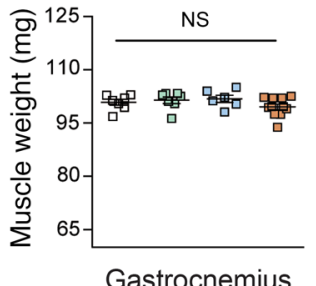

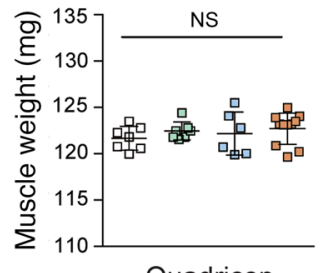

Quadricep

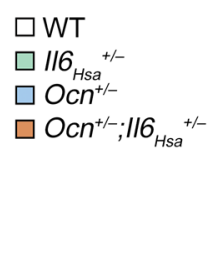

C
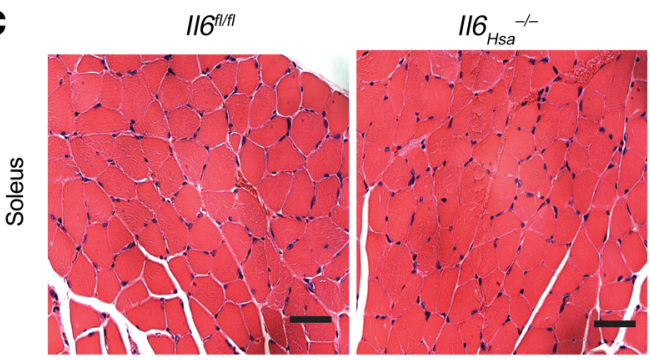

E

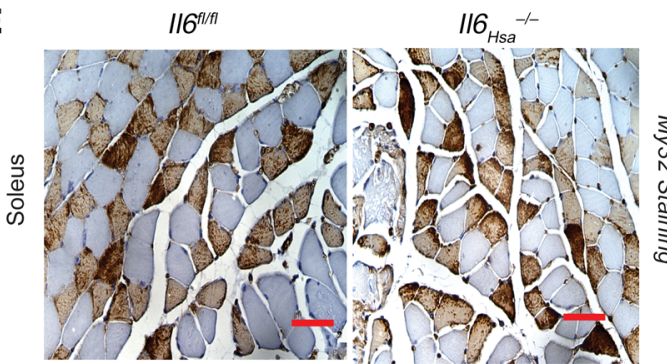

H

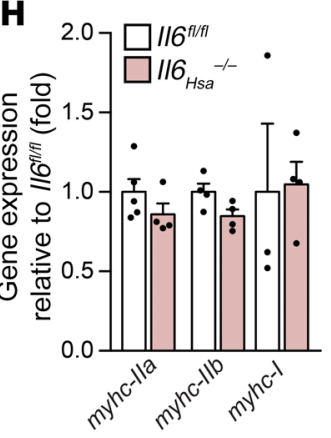

D
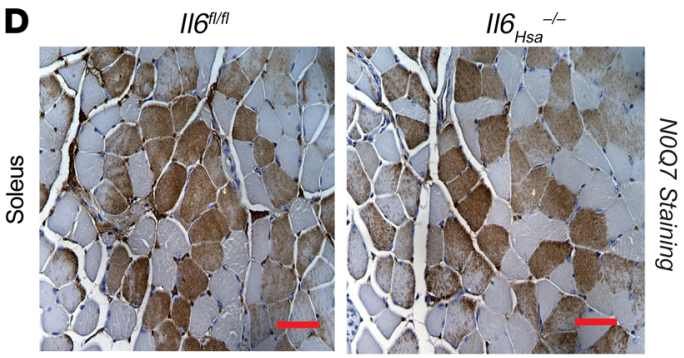

F

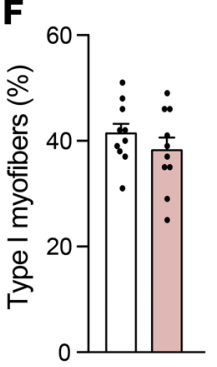

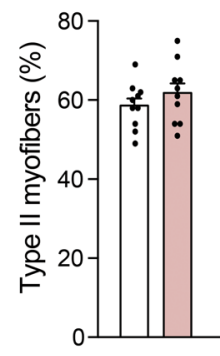

G
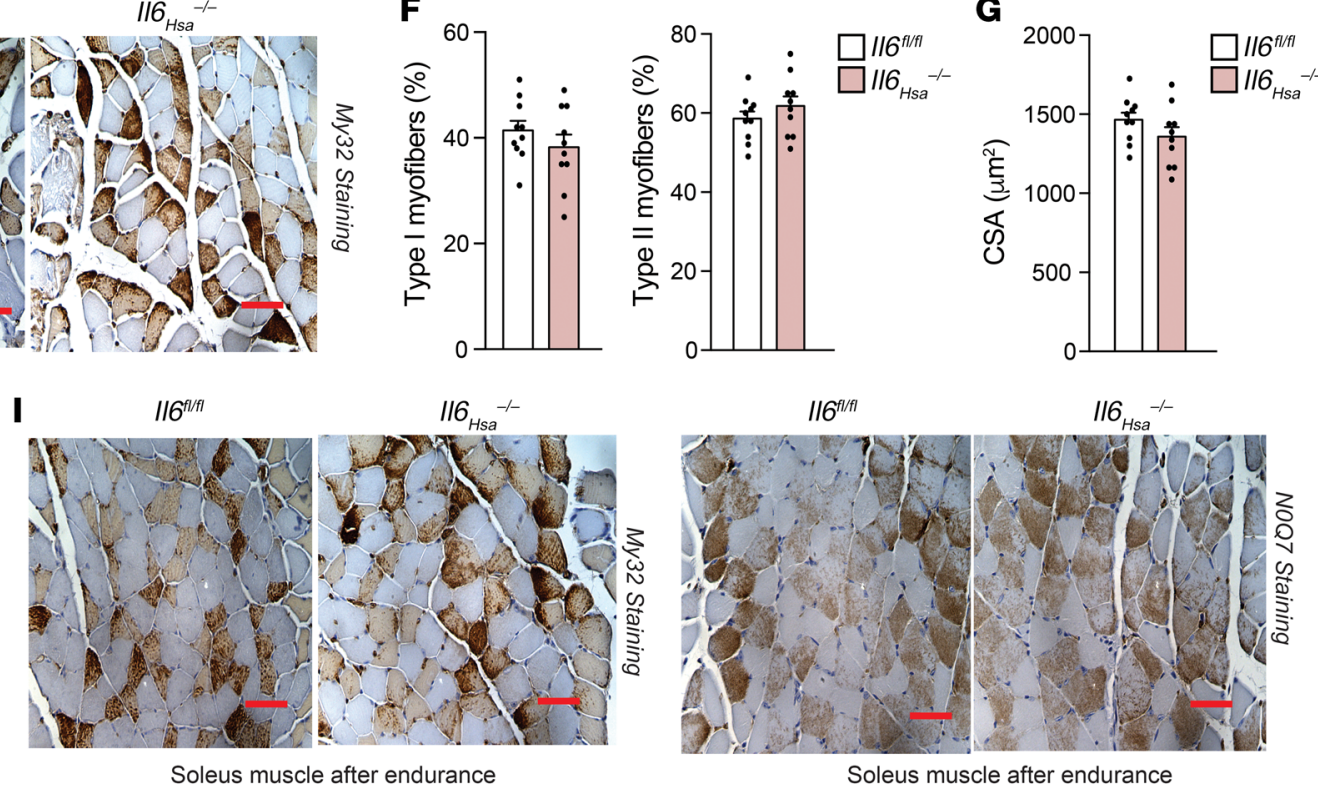

Soleus muscle after endurance

Figure 4. mIL-6 contributes to the maintenance of muscle mass in an osteocalcin-independent manner. (A) Weight of hind limb muscles of 3-month-old female $1 / 6_{\mathrm{Hsa}^{--}}$and control littermates, $n=6-11$. (B) Weight of hind limb muscles in 3-month-old $\mathrm{OCn}^{+/-} \| 16_{\mathrm{HsO}}{ }^{+/-}$compound mutant mice and control littermates (control group includes WT, Ocn ${ }^{+/-}$, and $I / 6_{H s a}{ }^{+/-}$mice), $n=5-7$. (C) Representative H\&E staining of soleus muscle fibers in 3-month-old female $I / 6_{H s a}{ }^{-/}$ and control littermates. Scale bars: $100 \mu \mathrm{m}$. (D and E) Representative histology with (D) N0Q7 (MHC I slow twitch fibers) and (E) MY32 (MHC II fast twitch fibers) staining of soleus muscle of 3-month-old female $1 / 6_{\mathrm{Hsa}}{ }^{-1-}$ and control littermates. Scale bars: $100 \mu \mathrm{m}$. (F) Distribution of type 1 and type II myofibers in 3-month-old female $1 / 6_{\mathrm{Hsa}^{-}}{ }^{-/-}$and control littermates. Scale bars: $100 \mu \mathrm{m}$. (G) Measurement of cross-sectional area (CSA) of muscle fibers in 3-month-old female $1 / 6_{H s s}{ }^{-1-}$ and control littermates. (H) Expression of myosin heavy chain genes in gastrocnemius muscle measured by qRT-PCR. (I) Representative histology with NOQ7 and MY32 staining of soleus muscle after endurance exercise in 3-month-old female $/ 16_{\mathrm{Hsa}^{-/-}}$and control littermates. Scale bars: 100 $\mu \mathrm{m}$. These results are representative of 3 independent experiments. Data shown in $\mathbf{A}, \mathbf{F}$, and $\mathbf{G}$ were analyzed by 2 -tailed unpaired $t$ test and data in $\mathbf{B}$ and H by 1-way ANOVA followed by Tukey's post hoc test. Data are presented as the mean \pm SEM. ${ }^{*} P<0.05$. 
mass. Indeed, if mIL-6 and osteocalcin were in the same genetic pathway controlling exercise capacity, compound double heterozygous $\mathrm{Ocn}^{+/-} \mathrm{Il}-6 \mathrm{Hsa}{ }^{+-}$mice should exhibit the same deficit in exercise capacity as the one observed in $\mathrm{Il6}_{\mathrm{Hsa}}{ }^{-/-}$or $\mathrm{Ocn}^{-/-}$mice, whereas the single heterozygous mice would run normally. As shown in Figure 3G, this is exactly what was observed. However, and as importantly, muscle mass was not decreased in any type of muscle in $\mathrm{Ocn}^{+/-} \mathrm{Il}-6 \mathrm{Hsa}^{+/-}$mice (Figure 4B). These data indicate on the one hand that the mIL- 6 regulation of muscle mass and muscle function occurs through different mechanisms. On the other hand, they show that mIL-6's regulation of muscle mass does not account for the mIL- 6 regulation of exercise capacity.

To ascertain whether there are any abnormalities in type I or type II muscle fiber frequency in the absence of mIL-6, we used 2 methods. First, we performed immunohistochemistry for either the slow myosin heavy chain (type I) or the fast myosin heavy chain (type II) and, second, we analyzed gene expression in soleus muscle of myosin heavy chain isoform 2a ( $\mathrm{MyHC}-\mathrm{II} a)$ and 2b (MyHC$I I b)$. In both cases there were no detectable differences in the abundance of type I or type II fibers in $\mathrm{Il}_{\mathrm{Hsa}}{ }^{-/-}$and control soleus muscles (Figure 4, C-E and H). Likewise, a histological analysis of the soleus muscle in 3-month-old $\mathrm{Il6}_{\mathrm{Hsa}}^{-1-}$ mice and control littermates did not reveal any significant difference in myofiber cross-sectional area (CSA) between $\mathrm{Il6}_{\mathrm{Hsa}}{ }^{-1-}$ and control soleus muscles (Figure 4G).

IL-6 favors osteoclastogenesis by signaling in osteoblasts. Any effort to unravel the cellular pathway whereby mIL- 6 enhances exercise capacity during endurance exercise must start with the identification of the cell type in which mIL-6 signals in bone. For that purpose, we relied on a classical coculture assay between osteoblasts and bone marrow-derived osteoclast progenitor cells that were obtained from mice of different genotypes (19). IL-6 exerts its biological activities through 2 molecules, IL-6R and the glycoprotein gp130, the signal transducing partner of IL-6R (20). Because gp130 contributes to the signaling of other cytokines, defining the importance of IL- 6 signaling in a given cell type in vivo requires the deletion of Il6rin that cell type.

All coculture experiments described below were performed in the presence of the soluble IL-6R (sIL-6R) (21). When Il6r-1osteoblasts were cocultured in the presence of IL-6 with $I l 6 r^{f / f l}$ osteoclast progenitor cells, the number of tartrate-resistant acid phosphatase-positive (TRAP-positive) osteoclasts and osteoclasts containing 4 or more nuclei were both significantly reduced compared with what was observed when both osteoblasts and osteoclast progenitor cells were derived from $I l 6 r^{f / f l}$ mice or

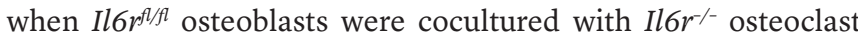
progenitor cells (Figure 5A). Furthermore, expression of markers of osteoclast differentiation or function, such as Dc-stamp, Atp6vdO2 (ATPase, $\mathrm{H}^{+}$transporting, V0 subunit D2), Clcn7 (chloride voltage-gated channel 7), and Acp5 (TRAP) was also significantly reduced in osteoclasts following coculture of $\mathrm{Il6r}^{-/}$osteoblasts with $I l 6 r^{f l / f l}$ osteoclast progenitor cells compared with what was observed when $I l 6 r^{f / f l}$ osteoblasts were cocultured with $I l 6 r^{f l / f l}$ or $\mathrm{Il}_{6} \mathrm{r}^{-1}$ osteoclasts progenitor cells (Figure 5B). We also performed

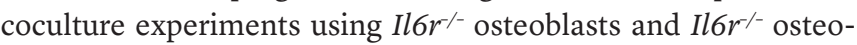
clast progenitor cells in the presence of sIL-6R. As shown in Figure $5 \mathrm{C}$, there was limited generation of $\mathrm{TRAP}^{+}$multinucleated osteoclasts in this experiment. This does not exclude the formal possibility that trans IL-6 signaling contributes to osteoclastogenesis in other experimental contexts.

Lastly, we cocultured $I l 6 r^{f / f l}$ osteoblasts or osteoclast progenitor cells that had been infected beforehand with an empty adenovirus or one expressing Cre recombinase. We verified in each case that infection with Cre achieved a deletion of $I l 6 r$ above 65\% (Supplemental Figure 2, A and B). Regardless of the presence or lack of IL- 6 in the culture medium, we found that when Il $6 r$ was deleted in osteoblasts the number of TRAP-positive multinucleated osteoclasts obtained was significantly decreased compared with what was observed in cocultures of $I l 6 r^{f / f l}$ osteoclast progenitor cells and $I l 6 r^{f / f l}$ osteoblasts infected with an empty adenovirus or one expressing Cre recombinase (Figure 5, D and E, and Supplemental Figure 2C). Of note, the RankL/Opg ratio was significantly higher in control coculture experiments than when IL-6R had been deleted from osteoblasts (Supplemental Figure 2D). Taken together, these experiments support the notion that in the conditions of these assays, IL- 6 acts primarily in cells of the osteoblast lineage to favor osteoclast differentiation.

IL-6 signaling in osteoblasts is needed to enhance exercise capacity during endurance exercise. To determine whether the ability of IL-6 signaling in osteoblasts to enhance osteoclast differentiation explains, at least in part, why IL-6 increases exercise capacity we crossed $I l 6 r^{f l / f l}$ mice with Ocn-Cre mice that delete genes in differentiated osteoblasts after birth (Figure 6A and refs. 22, 23). We chose this Cre driver mouse because we had shown earlier that mIL-6 does not influence exercise capacity in any noticeable manner before 3 months of age, and therefore it seems unlikely that IL-6 functions primarily in osteoblast progenitor cells. This manipulation resulted in an efficient deletion of Il6r in differentiated osteoblasts but not in osteoclasts or other cell types or other tissues tested in $I l 6 r_{O s b}{ }^{-1-}$ mice (Figure 6B). $\mathrm{Il} \mathrm{O}_{O s b}{ }^{-1-}$ mice were obtained at the expected Mendelian ratio, had a normal life span, and appeared overtly normal.

Despite a marked increase in circulating IL- 6 levels during

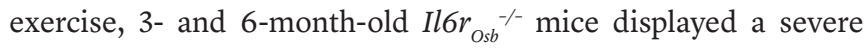
decrease in their ability to perform endurance exercise compared with control mice (Figure 6, C and D, and Supplemental Figure $3 \mathrm{~A})$. In contrast, mutant mice lacking IL-6R in myofibers only ran as well as control mice (Figure 6E). The deficit in exercise capacity observed in mice lacking IL-6 signaling in osteoblasts was not caused by a decrease in muscle mass or a low bone mass because muscle mass, bone mass, and markers of bone resorption at rest were all similar between $I l 6 r_{O s b}{ }^{-1-}$ and control mice, despite differences in markers of bone resorption during exercise (Supplemental Figure 3, B-D). These results indicate that IL-6 needs to signal in osteoblasts to favor exercise capacity.

Several lines of evidence indicate that the lack of secretion of bioactive osteocalcin is a major contributor to the deficit in exercise capacity of the $\mathrm{IlOr}_{\mathrm{Osb}}^{-1-}$ mice (Figure 6F). First, circulating osteocalcin levels did not increase in these mutant mice during endurance exercise (Figure 6, C-E). Second, compound heterozy-

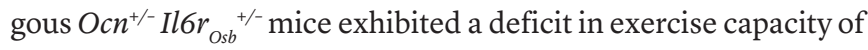

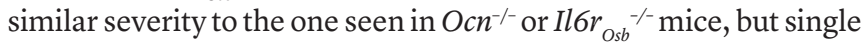
heterozygous mice did not (Figure 6G and ref. 13). Likewise, circulating osteocalcin levels did not increase in $\mathrm{Ocn}^{+/-} \mathrm{Il6r}_{\mathrm{Osb}}^{+--}$mice, as they did in control single heterozygous littermates during exercise 

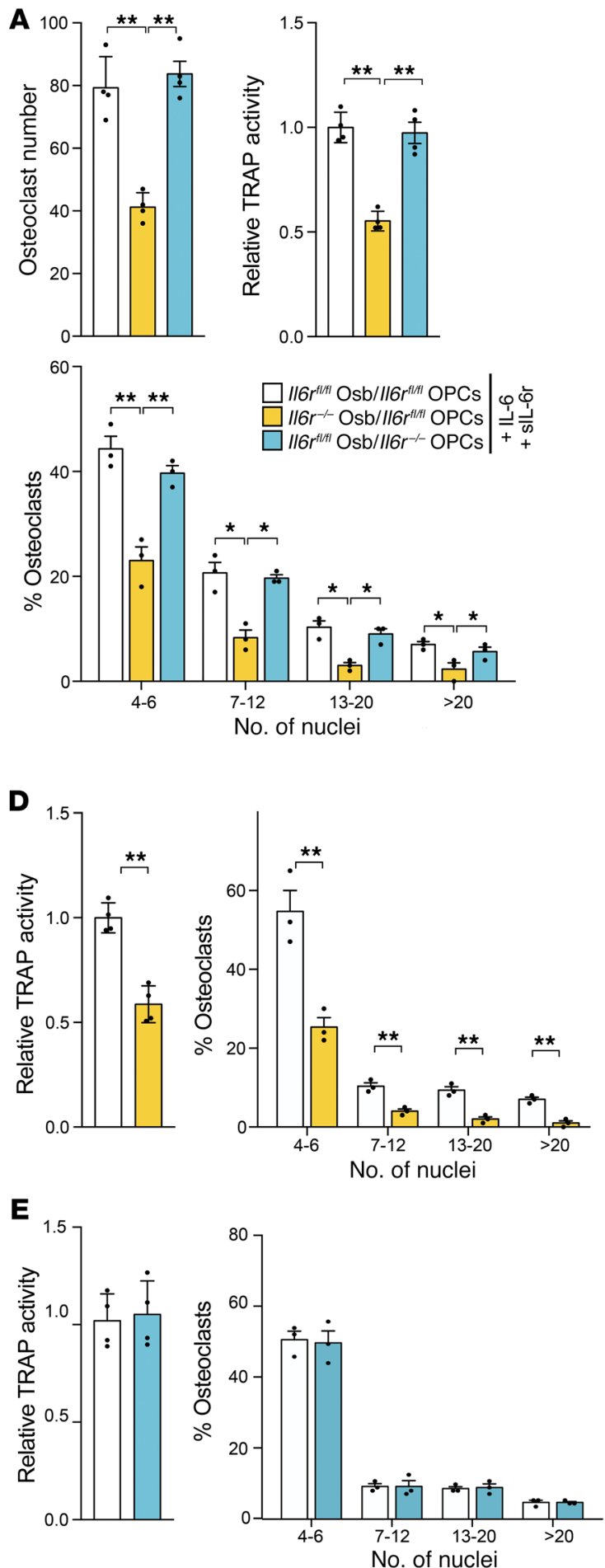

B
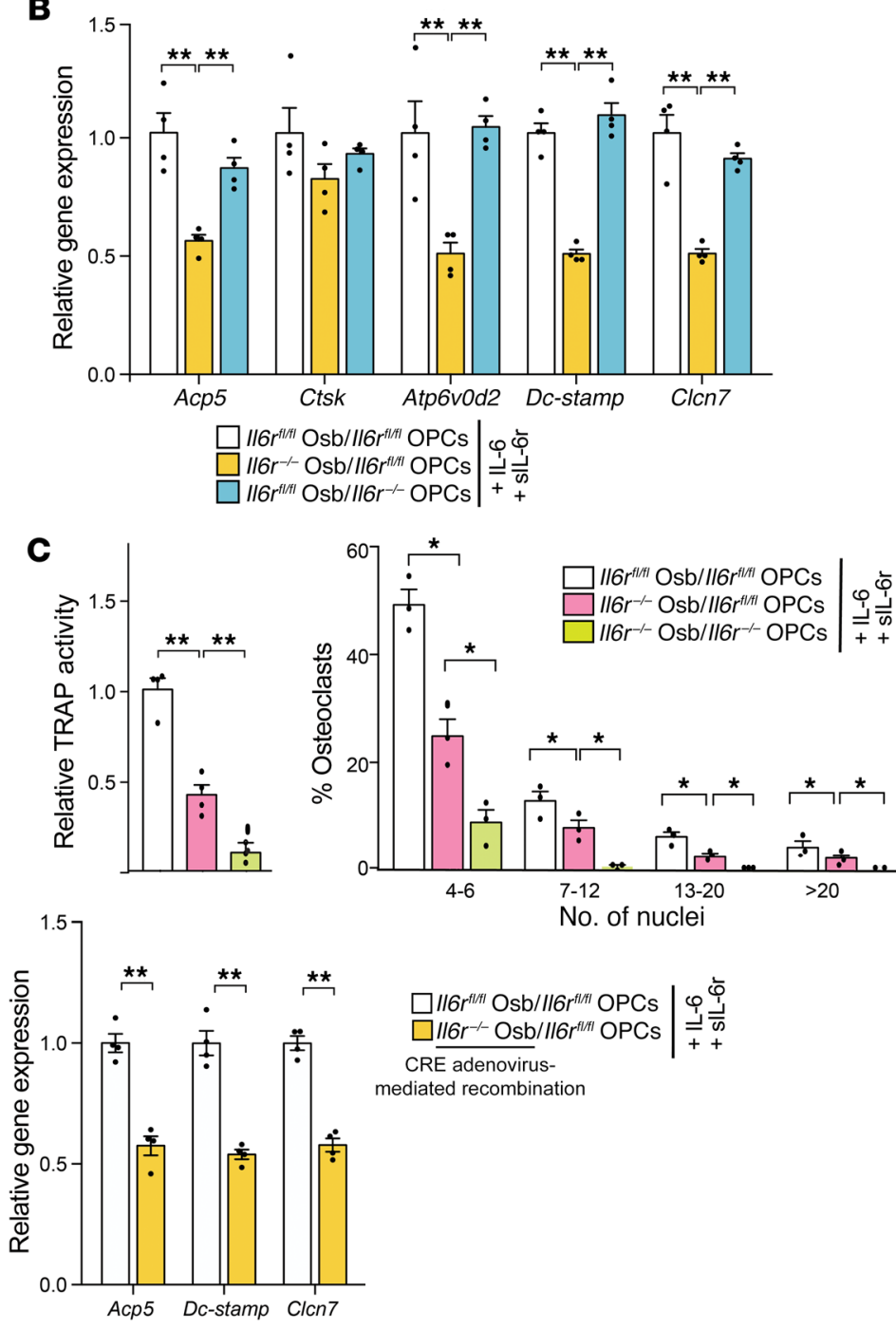

No. of nuclei

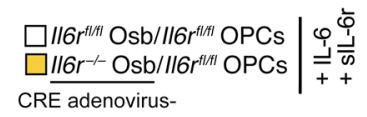

mediated recombination

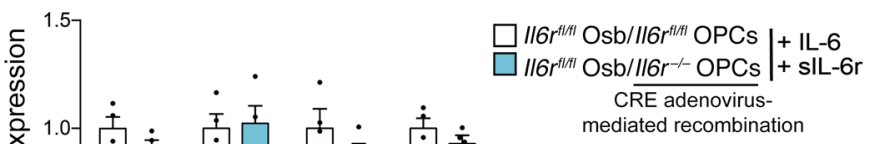

mediated recombination

Figure 5. IL-6 favors osteoclastogenesis by signaling in osteoblasts. (A and B) Coculture of $\| 6 \mathrm{r}^{f l / f l}$ osteoblasts with $/ / 6 \mathrm{r}^{\mathrm{fl} / \mathrm{fl}}$ osteoclast precursor cells (OPCs), $\| 6 r_{o s b}{ }^{-1-}$ osteoblasts with $\| \sigma^{f / / f l}$ OPCs, and $/ / 6 f^{f l / f l}$ osteoblasts with $/ / 6 r_{o s b}{ }^{-1-}$ OPCs in the presence of IL-6 and sIL-6r. (A) Quantification of the number of

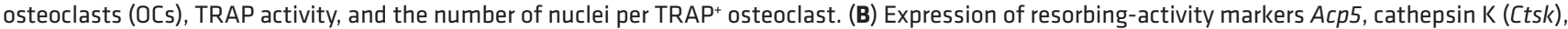
Atp6vOd2, Dc-stamp, and CIcn7 in mouse osteoclasts (WT OCs). (C) Coculture in the presence of IL-6 and sIL-6r of (i) $/ / 6 r^{f l / f l}$ osteoblasts with $/ / 6 r^{f l / f l}$ OPCs, (ii) $/ 16 r_{\text {osb }}{ }^{-1-}$ osteoblasts with $/ / 6^{f / / f l}$ OPCs, or (iii) $/ / 6 r^{-1-}$ osteoblasts with $\| 6 r_{o s b}{ }^{-1-}$ OPCs. TRAP activity and quantification of the number of osteoclasts. (D and E) $I L 6 r^{\prime-}$ osteoblasts were generated by infecting $/ / 6 r^{f / / f l}$ osteoblasts with adenovirus expressing Cre recombinase and coculturing with $/ / 6 r^{f / f f}$ osteoblasts in the absence or presence of IL-6 in the culture medium. Similarly, IL $6 r^{-1-}$ OPCs were generated by infecting $I / 6 r^{f l / f l}$ OPCs with adenovirus expressing Cre recombinase and coculturing with $/ / 6 r^{f l / f l}$ osteoblasts in the absence or presence of IL-6. (D) Quantification of TRAP activity, of the number of nuclei per TRAP ${ }^{+}$osteoclast, and gene expression of resorption markers (Acp5, Dc-stamp, and Clcn7). (E) Cocultures in the presence of IL-6 in the culture medium, quantification of TRAP activity, the number of nuclei per TRAP+ osteoclast, and expression of resorption markers (Acp5, Atp6v0d2, Dc-stamp, and Clcn7). These results are representative of 3 independent experiments with triplicate samples. Data in A-E were analyzed by 2-way ANOVA followed by Tukey's post hoc test; the relative TRAP activity data in $\mathbf{A}$ and $\mathbf{C}-\mathbf{E}$ were analyzed by 2 -tailed unpaired $t$ test. Error bars represent $\mathrm{SEM}$. ${ }^{*} P<0.05 ;{ }^{*} P<0.01$. 
A

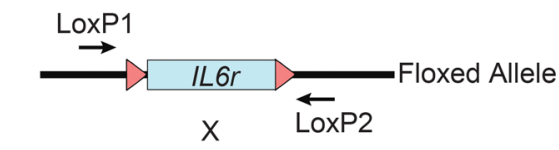

\begin{tabular}{ll|l|} 
Ocn Promoter & ATG & CRE \\
\hline
\end{tabular}

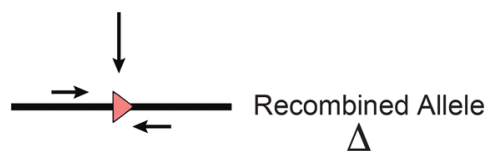

B

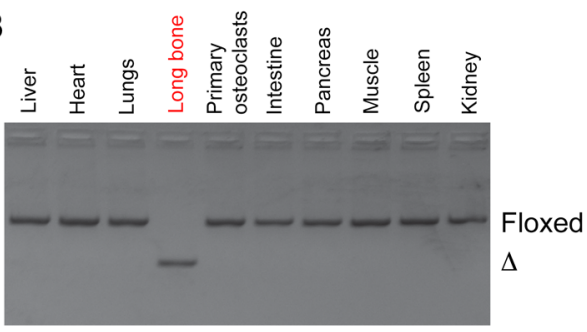

C

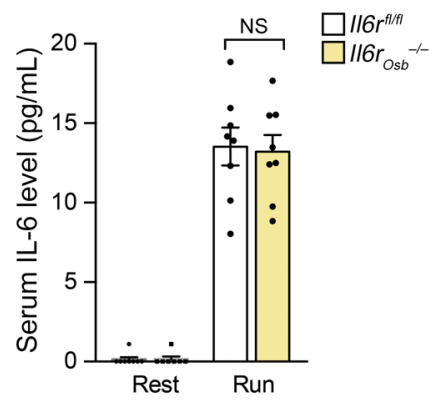

D

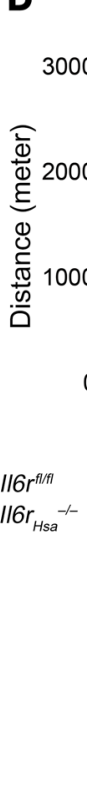

3 mo

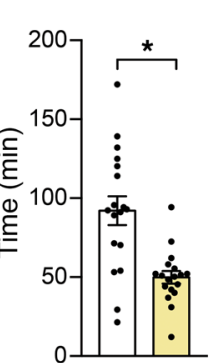

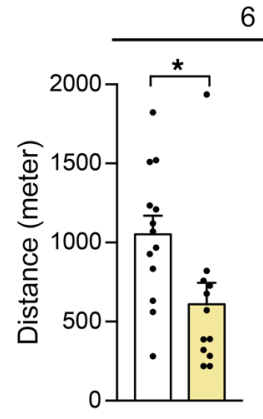

$6 \mathrm{mo}$
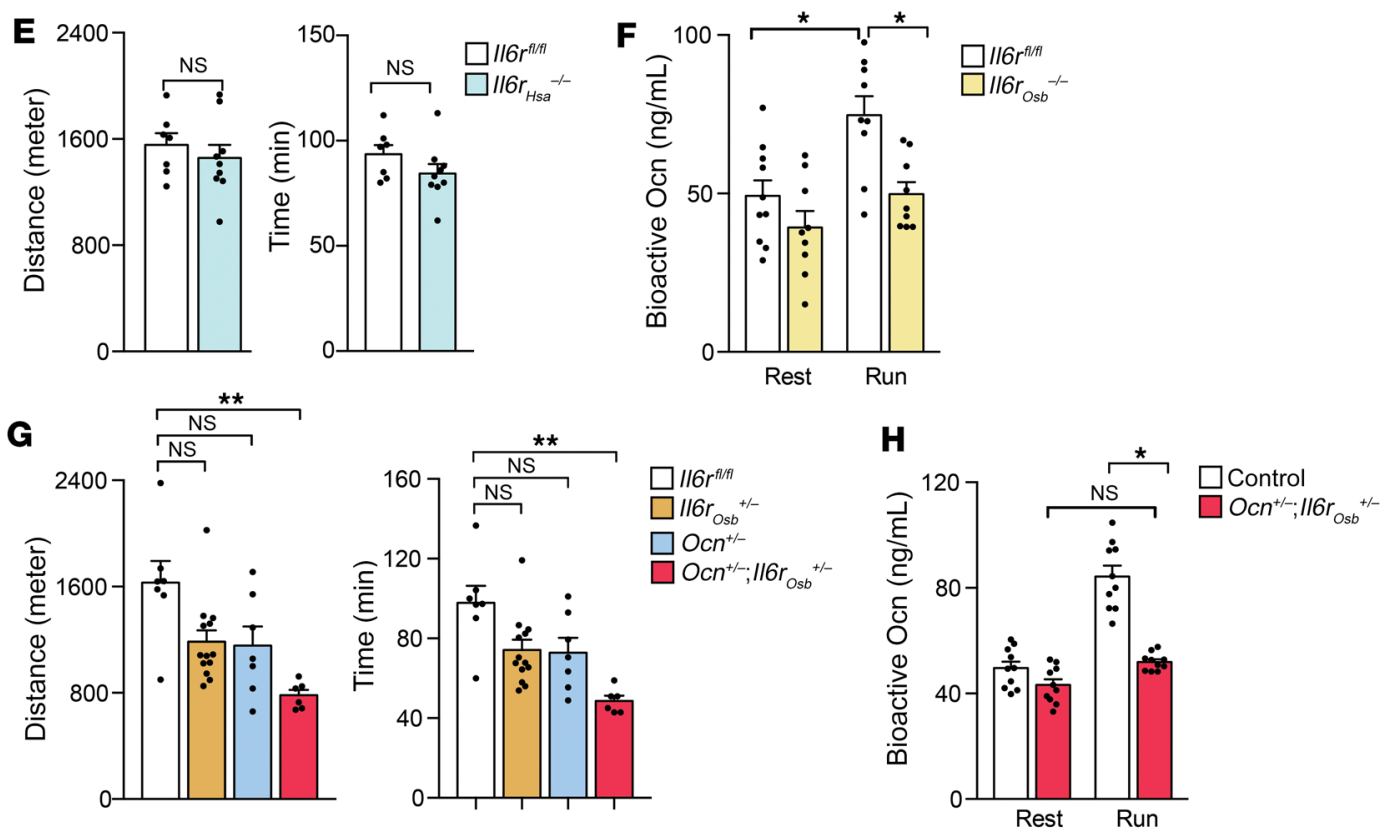

Figure 6. IL-6 signaling in osteoblasts is needed to enhance exercise capacity during endurance exercise. (A) Crossing of $/ / 6 \mathrm{r}^{\mathrm{fl} / f l}$ mice with $\mathrm{Ocn}$ - Cre mice to delete $/ / 6 r$ in differentiated osteoblasts after birth and generate $/ / 6 r_{0 s b}{ }^{-1-}$ mice. (B) Detection of $/ / 6 r$ deletion by PCR on genomic DNA isolated from various tissues of $\| 6 r_{0 s b}{ }^{-1-}$ mice. (C) Circulating IL-6 levels in 3-month-old $/ / 6 r^{f / / f l}$ and $/ / 6 r_{0 s b}{ }^{-1-}$ mice before and after exercise, $n=8$. (D) Performance during an

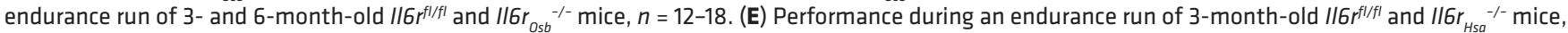
$n=7-9$. (F) Circulating Ocn levels at rest and after exercise in bones of 3-month-old $/ / 6 r^{f l / f l}$ and $/ / 6 r_{0 s b}{ }^{-1-}$ mice, $n=9-10$. (C) Performance during an endurance

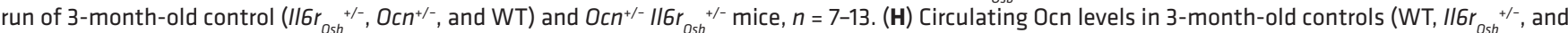
$\left.\mathrm{Ocn}^{+/-}\right)$and $\mathrm{OCn}^{+/-} \| \mathrm{Irr}_{\mathrm{Osb}}{ }^{+/-}$mice at rest and after exercise, $n=10$ each. These results are representative of 4 independent experiments. Data were analyzed by 1-way ANOVA followed by Tukey's post hoc test. Data presented as the mean $\pm \mathrm{SEM}$. ${ }^{*} P<0.05 ;{ }^{*} P<0.01$.

(Figure 6H). Third, an injection of IL-6 did not correct the deficit in exercise capacity in $\mathrm{Il}_{\mathrm{Osb}}{ }^{-1-}$ or in $\mathrm{Ocn}^{+/-} \mathrm{Il}_{\mathrm{Osb}}{ }^{+/}$mice even though it increased their circulating IL-6 levels (Figure 7, A-C, and Supplemental Figure 3E). Fourth and conversely, an injection of osteocalcin increased exercise capacity in both $\mathrm{IlGr}_{\mathrm{Osb}}{ }^{-1-}$ and $\mathrm{Ocn}^{+/-}$ Il $6 r_{O s b}{ }^{-1-}$ mice (Figure 7, D-G).

mIL-6 favors glucose uptake and catabolism in myofibers during exercise through osteocalcin. In the last set of experiments we explored whether the decrease in exercise capacity caused by the absence of either muscle-derived IL-6 or IL- 6 signaling in osteoblasts was secondary to a lack of substrate availability (24) or rather, as it is the case when osteocalcin signaling in myofibers is disrupted, to a decrease in substrate uptake and catabolism in muscle (13).

Glucose homeostasis assayed by glucose tolerance and insulin sensitivity tests and liver gluconeogenesis assayed by a pyruvate tolerance test were unaffected by the postnatal deletion of $I l 6$ 

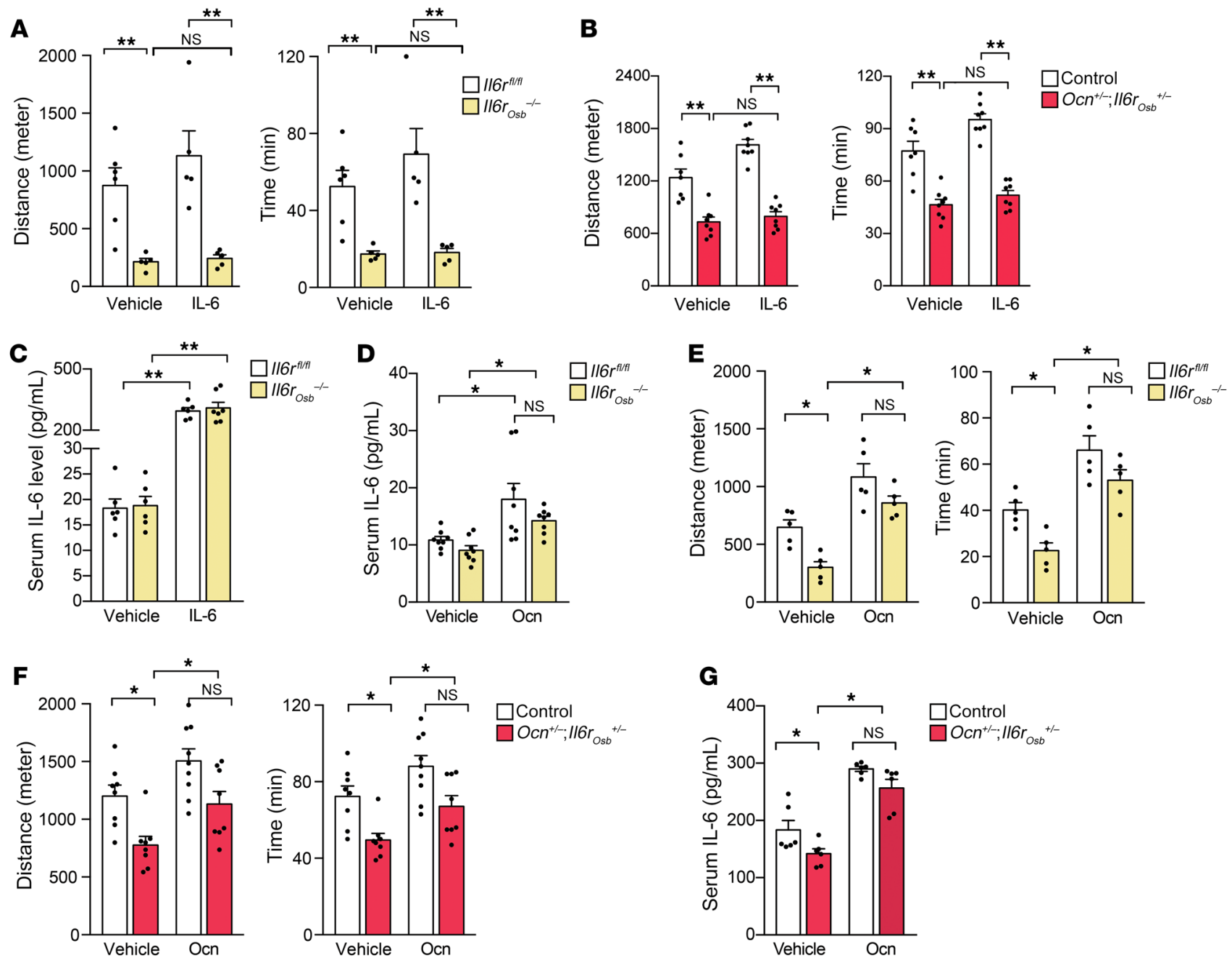

Figure 7. IL-6 signaling in osteoblasts is needed to enhance exercise capacity during endurance exercise. (A) Performance during an endurance run of 3-month-old $\| 6 \mathrm{r}^{\mathrm{fl} / \mathrm{fl}}$ and $\| 6 \mathrm{r}_{\mathrm{Osb}}{ }^{-1-}$ mice after an i.p. injection of IL-6 (3 $\left.\mathrm{ng} / \mathrm{g}\right), n=6$. (B) Performance during an endurance run of 3-month-old $I / 6 \mathrm{r}_{0 s b}{ }^{+/-} \mathrm{Ocn}^{+/-}$

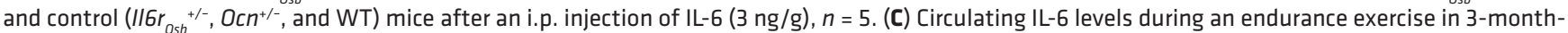
old $I / 6 r^{f l / f l}$ and $I / 6 r_{O s b}{ }^{-1-}$ mice after i.p. injection of IL-6 (3 ng/g), $n=6$. (D) Circulating IL-6 levels in 3-month-old $/ / 6 r^{f l / f l}$ and $/ / 6 r_{0 s b}{ }^{-1-}$ mice after i.p. injection of Ocn (120 ng/g), $n=8$. (E) Performance during an endurance exercise of 3-month-old $/ / 6 \mathrm{r}^{f / / f l}$ and $/ / 6 \mathrm{r}^{-1 /}$ mice treated with osteocalcin (Ocn, $120 \mathrm{ng} / \mathrm{g}$ ), $n=5$. (F) Performance during an endurance exercise of 3-month-old $/ 16 \mathrm{rr}_{\mathrm{Osb}}^{+/-} \mathrm{Ocn}^{+/-}, I / 6 \mathrm{rosb}^{+/-}, \mathrm{Ocn}^{+/-}$, and WT mice after an i.p. injection of Ocn (120 ng/g),

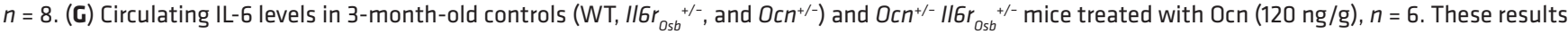
are representative of 4 independent experiments. Data were analyzed by 1-way ANOVA followed by Tukey's post hoc test. Results presented as the mean \pm SEM. ${ }^{*} P<0.05 ;{ }^{* *} P<0.01$.

from myofibers (Supplemental Figure 4, A-E). Likewise, expression in the liver of Pepck and G6pase that encode 2 key enzymes of liver gluconeogenesis was similar in $\mathrm{Il}_{\mathrm{Hsa}}{ }^{-1-}, \mathrm{Il}_{\mathrm{OSSb}}{ }^{-1-}$, and control mice before and after exercise, thus indicating that gluconeogenesis is not overtly decreased in the absence of mIL- 6 or of IL-6 signaling in osteoblasts (Supplemental Figure 4, F-I). The absence of any detectable decrease in substrate availability in mice lacking either mIL-6 or IL-6R in osteoblasts prompted us to test whether substrate uptake and catabolism in myofibers were decreased in the absence of mIL- 6 or IL- 6 signaling in osteoblasts.

Consistent with this notion, we found that glucose uptake, measured by the uptake of ${ }^{3} \mathrm{H}$-2-deoxyglucose $\left({ }^{3} \mathrm{H}-2-\mathrm{DG}\right)$, was decreased in oxidative muscles of both $\mathrm{IlO}_{\mathrm{Hsa}}{ }^{-1-}$ and $\mathrm{Il6r} \mathrm{Osb}^{-1-}$ mice compared with their respective control littermates (Figure 8, A and B). Moreover, expression of Pgma1 that is necessary for gly- colysis was decreased in oxidative muscles of $\mathrm{Il}_{\mathrm{Hsa}}{ }^{-1-}$ mice after exercise (Figure 8C). A similar decrease in glucose uptake was observed in $\mathrm{Ocn}^{+/-} \mathrm{Il}_{\mathrm{Hsa}}{ }^{+/-}$and $\mathrm{Ocn}^{+/-} \mathrm{IlGr}_{\mathrm{Osb}}{ }^{+-}$mice, further supporting the notion that osteocalcin mediates the positive effect of mIL-6 on glucose uptake in myofibers during exercise (Figure 8, $\mathrm{D}$ and $\mathrm{E})$. To further demonstrate that this is the case we injected osteocalcin or IL-6 into $\mathrm{Il}_{\mathrm{Hsa}}^{-1-}, \mathrm{Ilbr}_{\mathrm{Osb}}{ }^{-1-}, \mathrm{Ocn}^{+/-} \mathrm{Ilb}_{\mathrm{Hsa}}^{+}{ }^{+-}$, or $\mathrm{Ocn}^{+/-}$ $I l 6 r_{O s b}{ }^{+/-}$mice. Osteocalcin injections normalized glucose uptake in muscle in all mutant mouse strains regardless of whether they increased circulating IL-6 levels or not (Figure 8, F-I, Supplemental Figure 4, J-M, and Table 1). IL-6 injections increased circulating IL-6 levels in all mutant mouse strains and it corrected the deficit in glucose uptake only in $\mathrm{Il}_{\mathrm{Hsa}}^{-1-}$ and $\mathrm{Ocn}^{+/-} \mathrm{Il}_{\mathrm{Hsa}}^{+/-}$mice in which circulating osteocalcin levels increased after exercise, but not in $\mathrm{IlGr}_{\mathrm{Osb}}{ }^{-1-}$ or $\mathrm{Ocn}^{+/-} \mathrm{IlGr}_{\mathrm{Osb}}{ }^{+/-}$mice in which circulating osteo- 
A

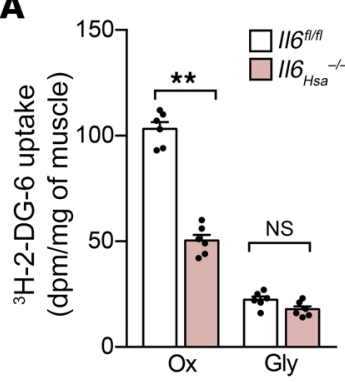

E
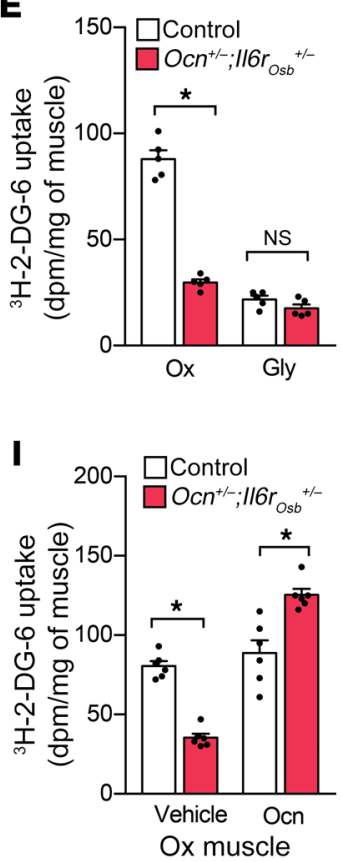

B

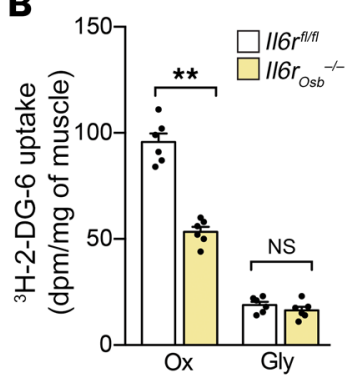

$\mathbf{F}$

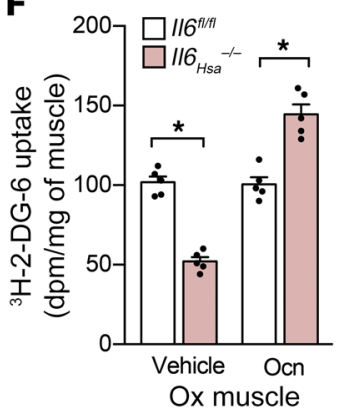

C

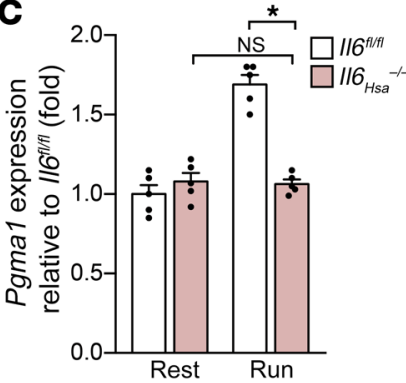

G

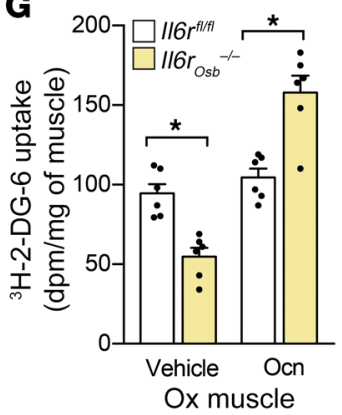

H
D
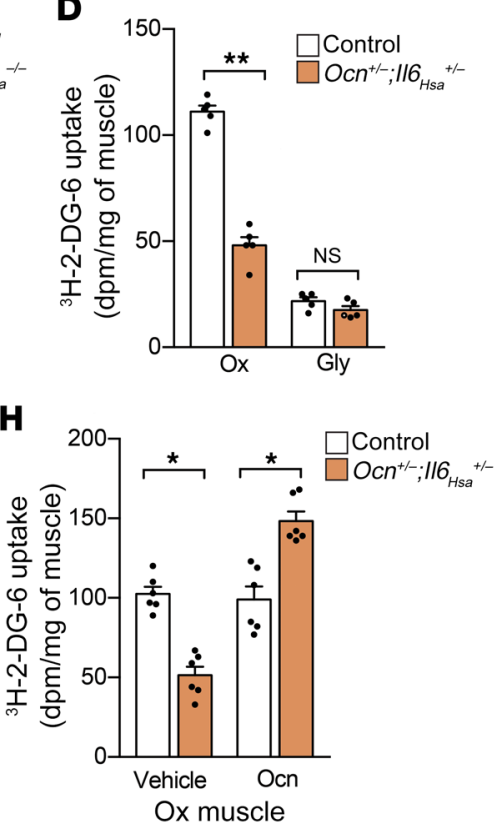
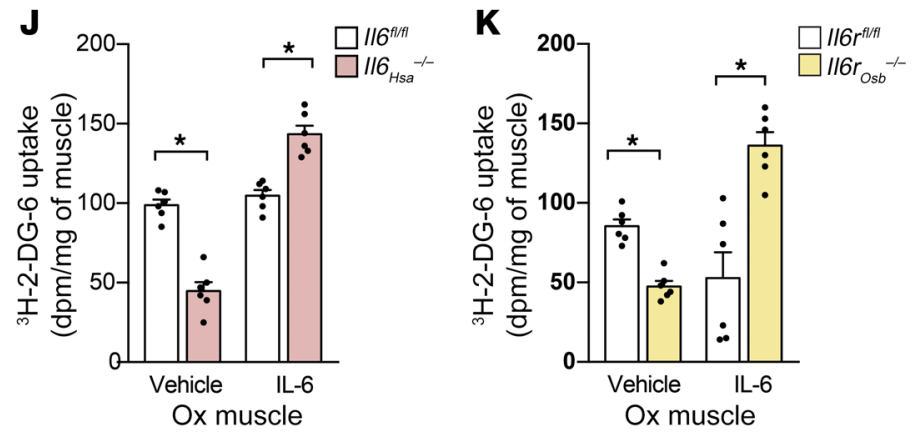

Figure 8. mIL-6 favors glucose uptake and catabolism in myofibers during exercise through osteocalcin. (A and B) Uptake of ${ }^{3} \mathrm{H}-2$-DC in glycolytic (Cly, white quadriceps) and oxidative (Ox, red quadriceps) muscle in 3-month-old mice. (A) $/ / 6^{f l / f l}$ versus $\| 6_{H s a}{ }^{-1-}$ and (B) $/ / 6 r^{f l / f l}$ versus $\| / 6 r_{0 s b}{ }^{-1-}$ mice after exercise, $n=6$. (C) Pgma1 expression at rest and after exercise in gastrocnemius muscle of 3-month-old $I / 6^{f / f f l}$ versus $I / 6_{H s a}{ }^{-1-}$ mice, $n=5$ (3 replicates for each mouse). (D and E) Uptake of ${ }^{3} \mathrm{H}-2-\mathrm{DC}$ in oxidative (Ox, red quadriceps) muscle in 3-month-old (D) controls $\left(1 / 6_{\mathrm{Hsa}}{ }^{+/-}\right.$and $\mathrm{Ocm}^{+/-}$) versus $\mathrm{Ocn}^{+/-} / 16_{\mathrm{Hsa}}{ }^{+/-}$, and (E) controls $\left(1 / 6 r_{\text {ssb }}{ }^{+/-}\right.$and $\left.\mathrm{OCn}^{+/-}\right)$versus $\mathrm{Ocn}^{+/-} / 16 \mathrm{r}_{\text {osb }}{ }^{+/-}$mice, $n=6$. (F-I) Uptake of ${ }^{3} \mathrm{H}-2-\mathrm{DC}$ in oxidative (Ox, red quadriceps) muscle after exercise in 3-month-old (F)

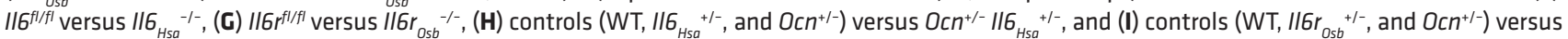
$\mathrm{Ocn}^{+/-} \| 6 \mathrm{O}{ }^{+1 /-}$ mice treated with osteocalcin (Ocn, $120 \mathrm{ng} / \mathrm{g}$ ) i.p., $n=6$. (J and $\mathbf{K}$ ) Uptake of ${ }^{3} \mathrm{H}-2-\mathrm{DC}$ in oxidative (Ox, red quadriceps) muscle after exercise in

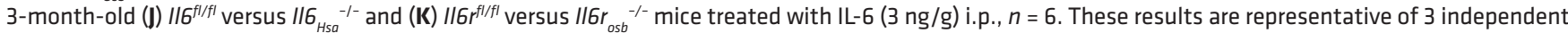
experiments. Data were analyzed by 1-way ANOVA followed by Tukey's post hoc test. Results presented as the mean \pm SEM. ${ }^{*} P<0.05$; ${ }^{* *} P<0.01$.

calcin levels did not increase following an IL-6 injection (Figure 8 , J and $\mathrm{K}$, and Table 1$)$.

mIL-6 favors fatty acid uptake and catabolism in myofibers during exercise through osteocalcin. Expression in white adipose tissue of hormone-sensitive lipase ( $H s l$ ) and adipose triglyceride lipase (Atgl) that are necessary for lipolysis was similar in $\mathrm{Il}_{\mathrm{Hsa}}^{-1-}$ and control mice before and after exercise (Supplemental Figure 5A). Circulating levels of nonesterified fatty acids (NEFAs) and triglycerides were either normal or increased in $\mathrm{IlO}_{\mathrm{Hsa}}{ }^{-1-}$ and $\mathrm{Ilbr}_{\mathrm{Osb}}{ }^{-1-}$ mice compared with their control littermates before and after exercise (Figure 9, A and B, and Supplemental Figure 5, B and C). Circulating glucagon and glycerol levels were also unchanged, as was the expression of mitochondrial biogenesis markers in $\mathrm{Il}_{\mathrm{Hsa}}{ }^{-1-}$ and control mice before and after exercise (Supplemental Figure 5, D-F).

We also studied fatty acid (FA) uptake and catabolism. As seen in mice lacking osteocalcin signaling in myofibers (13), expression of Fatp1, which facilitates the uptake of long-chain FAs into cells and of $C p t 1 b$, which promotes their transports across the mitochondrial membrane (25), was markedly decreased in muscle of $\mathrm{Il}_{\mathrm{Hsa}}{ }^{-1}$, $\mathrm{Ocn}^{+/-} \mathrm{Ilb}_{\mathrm{Hsa}}^{+-}, \mathrm{IlGr}_{\mathrm{Osb}}{ }^{-1-}$, and $\mathrm{Ocn}^{+/-} \mathrm{IlGr}_{\mathrm{Osb}}{ }^{+/-}$mice compared with their respective controls after exercise (Figure 9, C-F). Furthermore, phosphorylation at Ser563 of HSL, the enzyme that hydrolyzes intracellular triglycerides into free FAs, was decreased in

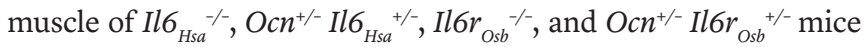
(Figure 9, G-J). Collectively, these results support the notion that mIL- 6 favors adaptation to exercise by promoting the production by bone of bioactive osteocalcin that in turn increases the uptake and catabolism of nutrients, i.e., glucose and FAs, in myofibers.

\section{Discussion}

This study was designed to address 2 questions related to IL-6 regulation of exercise capacity. The first one, prompted by the pleio- 


\section{Table 1. Summary of effects of intraperitoneal injection of osteocalcin or IL-6 during endurance run in different mouse strains}

\begin{tabular}{|c|c|c|c|c|c|}
\hline Genotype & Treatment & Glucose Uptake & Ocn Levels & IL-6 Levels & Run \\
\hline \multirow[t]{3}{*}{$1 / 6_{H s a}^{-1-}$} & Vehicle & Low & $\downarrow$ & $\downarrow$ & $\downarrow$ \\
\hline & Ocn $(120 \mathrm{ng} / \mathrm{g})$ & Rescued & $\uparrow$ & $\uparrow$ & $\uparrow$ \\
\hline & IL-6 (3 ng/g) & Rescued & $\uparrow$ & $\uparrow$ & $\uparrow$ \\
\hline \multirow[t]{3}{*}{$116 r_{0 s b}^{-1-}$} & Vehicle & Low & $\downarrow$ & $\downarrow$ & $\downarrow$ \\
\hline & Ocn $(120 \mathrm{ng} / \mathrm{g})$ & Rescued & $\uparrow$ & $\uparrow$ & $\uparrow$ \\
\hline & IL-6 (3 ng/g) & Low & $\rightarrow$ & $\uparrow$ & $\uparrow$ \\
\hline \multirow{3}{*}{$\mathrm{OCn}^{+/-} \| \mathbf{K}_{\mathrm{Hsa}}^{+/-}$} & Vehicle & Low & $\downarrow$ & $\downarrow$ & $\downarrow$ \\
\hline & Ocn $(120 \mathrm{ng} / \mathrm{g})$ & Rescued & $\downarrow$ & $\uparrow$ & $\uparrow$ \\
\hline & IL-6 (3 ng/g) & Rescued & $\uparrow$ & $\uparrow$ & $\downarrow$ \\
\hline \multirow[t]{3}{*}{$\mathrm{OCn}^{+/-} \| \mathrm{Ir}_{0 \mathrm{sb}}^{+/-}$} & Vehicle & Low & $\downarrow$ & $\downarrow$ & $\downarrow$ \\
\hline & Ocn $(120 \mathrm{ng} / \mathrm{g})$ & Rescued & $\uparrow$ & $\uparrow$ & $\uparrow$ \\
\hline & IL-6 (3 ng/g) & Low & $\rightarrow$ & $\uparrow$ & $\downarrow$ \\
\hline
\end{tabular}

tropic expression of Il6, was to determine to what extent does muscle contribute to the surge of circulating IL-6 levels during exercise. The second one was to define the cellular and molecular mechanisms used by IL- 6 and/or mIL- 6 to increase exercise capacity.

Addressing the first question through genetic means in the mouse established that the majority of IL-6 molecules detected in general circulation during an endurance exercise originate from myofibers. The analysis of $\mathrm{Il}_{\mathrm{Hsa}}^{-{ }^{--}}$mice revealed several other aspects of IL- 6 biology of great importance for our understanding of the regulation of exercise capacity during endurance exercise. The first one is that mIL- 6 is necessary to achieve optimal exercise capacity. Second, mIL- 6 is also responsible for the majority of the increase in circulating osteocalcin levels that occurs during endurance exercise. Third, the fact that an injection of osteocalcin largely rescued the deficit in exercise capacity in both $\mathrm{Il}_{\mathrm{Hs}}{ }^{-/-}$and $\mathrm{Il6}_{\mathrm{Hsa}}{ }^{+/-} \mathrm{OCn}{ }^{+-}$mice suggested that osteocalcin mediates, at least in part, the ability of IL- 6 to increase exercise capacity. This assumption does not exclude the possibility that IL-6 might increase exercise capacity through additional means.

A less anticipated observation, related to muscle biology, that the analysis of $\mathrm{IlO}_{\mathrm{Hsa}}^{--}{ }^{--}$mice revealed is that mIL-6 is necessary to maintain muscle mass in adult mice. A question this finding raised is whether this decrease in muscle explains, at least in part, the decrease in endurance exercise that the $\mathrm{Il6}_{\mathrm{Hsa}}{ }^{-1-}$ mice experience. To address this question we relied on genetic epistasis, reasoning that if indeed the decrease in muscle function was secondary to the decrease in muscle mass then compound heterozygous mice lacking 1 allele of Ocn and 1 allele of Il6 in muscle should experience either both phenotypes, i.e., a decrease in muscle mass and muscle function, or none of them. That $\mathrm{Il6}_{\mathrm{Hsa}}{ }^{+/-} \mathrm{Ocn}^{+/-}$mice exhibit the same deficit in exercise capacity as $I l 6_{\mathrm{Hsa}}{ }^{-/-}$mice but have a normal muscle mass indicates that the decrease in muscle function is not a consequence of the decrease in muscle mass. Of note, the decrease in muscle function during aerobic exercise also develops in $\mathrm{Ocn}^{--}$mice in the face of a normal muscle mass, further indicating that muscle function is not dependent on muscle mass $(13,26)$. This has important implications for the treatment of the decrease in muscle function in various sarcopenic syndromes. Of note, we did not detect any overt histological changes in muscle of mice lacking mIL-6 or its receptor in osteoblasts.

Underscoring its biological importance, we note that the crosstalk between IL-6 regulation of exercise capacity and osteocalcin extends beyond the mouse because an injection of osteocalcin increases circulating IL-6 levels in nonhuman primates, as it does in rodents (13). Furthermore, circulating osteocalcin also increased following a 12-weeklong training intervention in humans, as it does in mice after a single bout of endurance exercise. Importantly for our purpose, this increase in circulating osteocalcin levels in humans was dependent on IL-6 signaling, as is the case in the mouse. This conservation of the interplay between exercise, osteocalcin, and IL- 6 was a further incentive to decipher the mechanisms whereby IL-6 regulates adaptation to exercise and the release of osteocalcin from bone.

We first established through cell-based assays that IL-6 signals primarily in osteoblasts to favor osteoclast differentiation and the release of bioactive osteocalcin. These results, obtained using genetic approaches in cell culture, are in full agreement with previous reports (19) but do not exclude the possibility that IL-6 may also act on osteoclast progenitor cells at a level we could not detect in our experimental conditions. They also do not exclude the possibility that the source of IL-6 signaling in osteoblasts might be the resorbed extracellular matrix rather than the general circulation. Regardless, these observations were an obvious incentive to analyze the consequences for exercise capacity of deleting IL-6R from osteoblasts. We used for that purpose a Cre driver mouse that deletes genes in differentiated osteoblasts because the deficit in exercise capacity observed in the absence of mIL- 6 does not develop before 3 months of age, thus suggesting it is not the result of a developmental process (22). The inactivation of IL-6 signaling in differentiated osteoblasts resulted in a severe deficit in exercise capacity that develops in the face of a normal muscle mass. These results contrast with those obtained after the deletion of the IL-6R in myofibers because this latter deletion does not hamper in any measurable way the ability of mice to perform an endurance exercise. They also indicate that muscle mass does not necessarily predict muscle function during exercise.

Importantly, $I l 6 r_{O s b}{ }^{-1-}$ mice did not experience an increase in circulating osteocalcin during exercise and their deficit in exercise capacity was corrected by osteocalcin but not by IL-6. Taken together, these results identify osteocalcin, a hormone known to improve muscle function during exercise (13), as a major mediator of this function of IL-6 signaling in osteoblasts. Although we did not detect in our experimental setting any measurable influence of mIL- 6 in the generation of glucose and FAs, the 2 substrates of myofibers during exercise, we do not exclude the possibility that such a mechanism of action may also contribute to mIL-6's ability to enhance exercise capacity at a level that was not detected by the assays we used (9).

The cross-regulation between IL- 6 and osteocalcin presented in an earlier study (13) and this study raises the question of which 
A

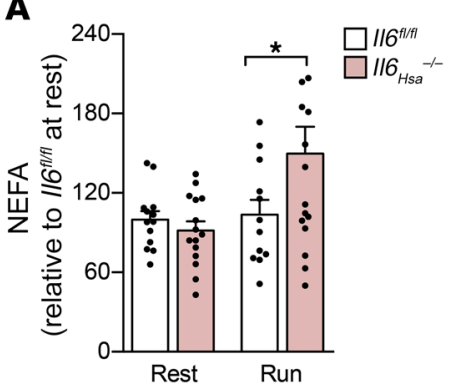

B

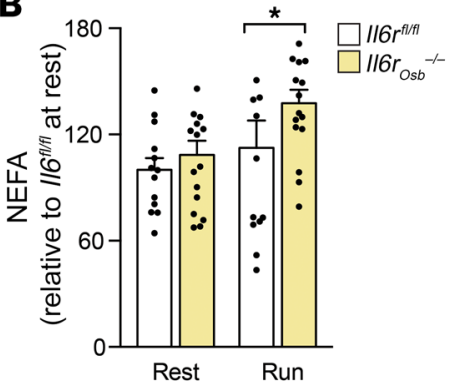

C

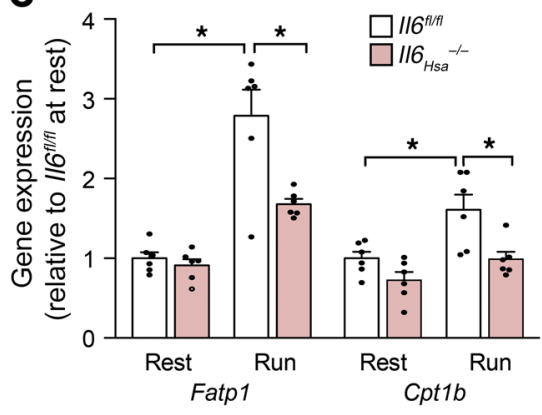

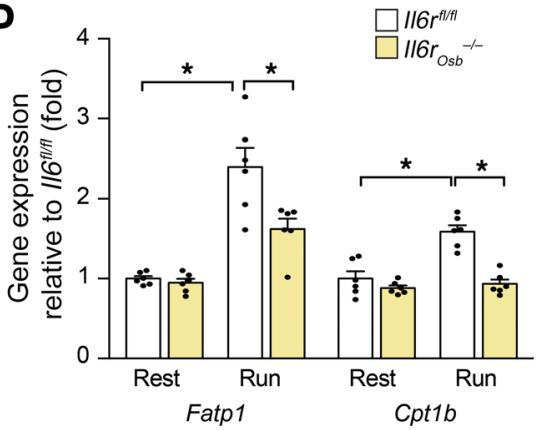

E

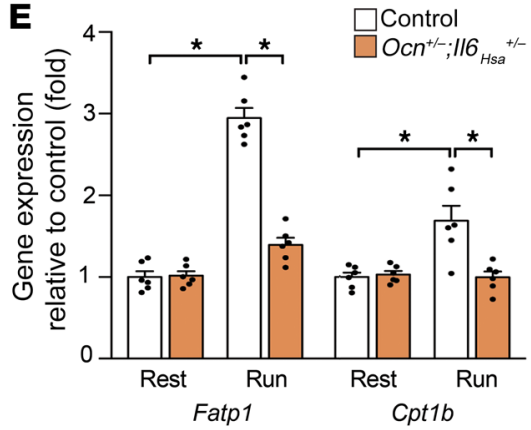

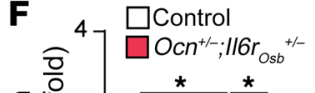

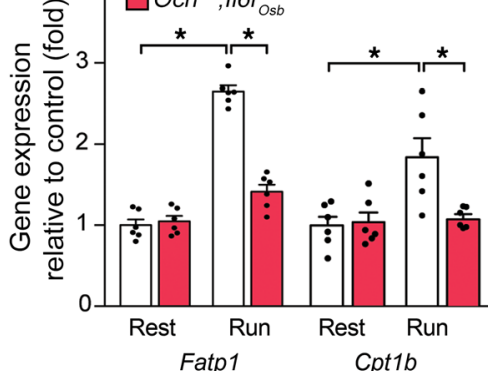

G

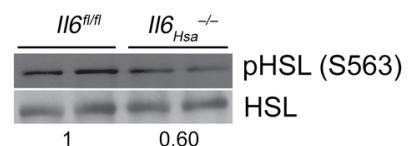

$\mathbf{H}$

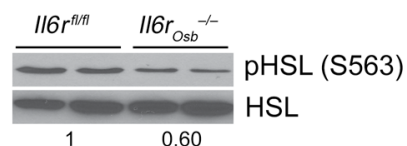

I

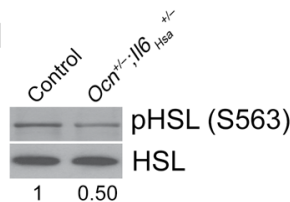

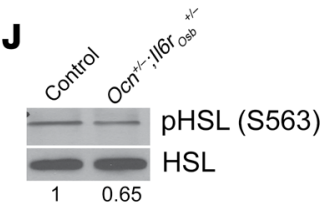

Figure 9. mIL-6 favors fatty acid uptake and catabolism in myofibers during exercise through osteocalcin. (A and B) Circulating NEFA levels at rest and after exercise in 3-month-old $I / 6_{H s a^{-1}}$ and $I / 6 r_{0 s b}{ }^{-1-}$ mice and their respective controls. (C-F) Expression of Fatp1 and Cpt $1 b$ at rest and after exercise in gas-

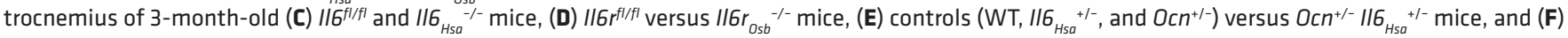

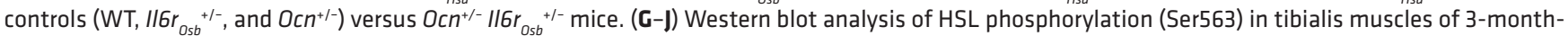

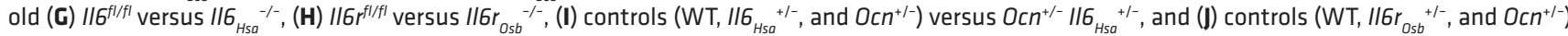
versus $\mathrm{Ocn}^{+/-} \| \mathrm{Irr}_{\text {Osb }}{ }^{+/-}$mice after exercise. These results are representative of 3 independent experiments. Data in A-E were analyzed by 1-way ANOVA followed by Tukey's post hoc test. Results presented as the mean \pm SEM. ${ }^{*} P<0.05$.

arm of this cross-regulation occurs first. Although this is a difficult question to address in vivo, the fact that osteocalcin acts directly on myofibers to upregulate Il6 expression, whereas IL-6 needs to trigger a multistep pathway involving gene expression in osteoblasts, osteoclast differentiation, and eventually release bioactive osteocalcin, suggests that the bone-to-muscle arm of this crosstalk is what initiates it.

The muscle-to-bone-to-muscle pathway favoring exercise capacity described here (Figure 10) complements the previously described bone-to-muscle pathway that is also necessary to increase exercise capacity (13). Together, these 2 studies propose a new perspective on how exercise capacity is regulated by distinguishing between muscle mass and muscle function and by identifying bone via the hormone osteocalcin as a focal regulator of exercise capacity. They also provide credence to the recently proposed notion that bone might have evolved as a tool to escape danger (27).

Going forward, 2 questions will need to be addressed. First, could osteocalcin signaling in myofibers be harnessed to reverse the course of sarcopenia in clinically relevant animal models of this disease? In the long run these experiments may pave the way to harness osteocalcin signaling in muscle in order to improve muscle function in sarcopenic patients. Second, if osteocalcin emerges as a central regulator of exercise capacity it may also signal in other organs implicated in this process.

\section{Methods}

Primate studies. Adult female rhesus monkeys (Macaca mulatta) weighing 7 to $10 \mathrm{~kg}$ were individually housed in standard primate cages at the large animal facility, National Institute of Immunology, New Delhi, India. During the experimentation period, the animal rooms received continuous fresh $5-\mu \mathrm{m}$-filtered air ranging from $22^{\circ} \mathrm{C}-28^{\circ} \mathrm{C}$ and $17^{\circ} \mathrm{C}-21^{\circ} \mathrm{C}$, maximum and minimum, respectively. Monkeys were injected at 1000 hours with recombinant human osteocalcin at the dose of $13.5 \mathrm{ng} / \mathrm{g}$ and blood samples were obtained by femoral venipuncture in Vacutainer blood collection tubes at different time points after injection. Blood was allowed to collect at room temperature, and serum was collected after centrifugation in aliquots and stored at $-80^{\circ} \mathrm{C}$ until analyzed.

Mouse studies. Il $6^{A / f l}$ and $\mathrm{HSA}{ }_{\text {MerCreMer }}\left(\mathrm{HSA}{ }_{M C M}\right.$ ) mice (Jackson Laboratory) were on the C57BL/6 genetic background. $\mathrm{Ocn}^{-/}$mice were

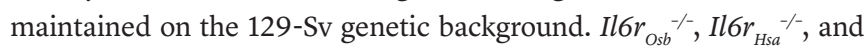
$\mathrm{Il}_{\mathrm{Hsa}}+/-\mathrm{OCn}^{+/}$mice were maintained on the $129-\mathrm{Sv} / \mathrm{C} 57 \mathrm{BL} / 6$ mixed 


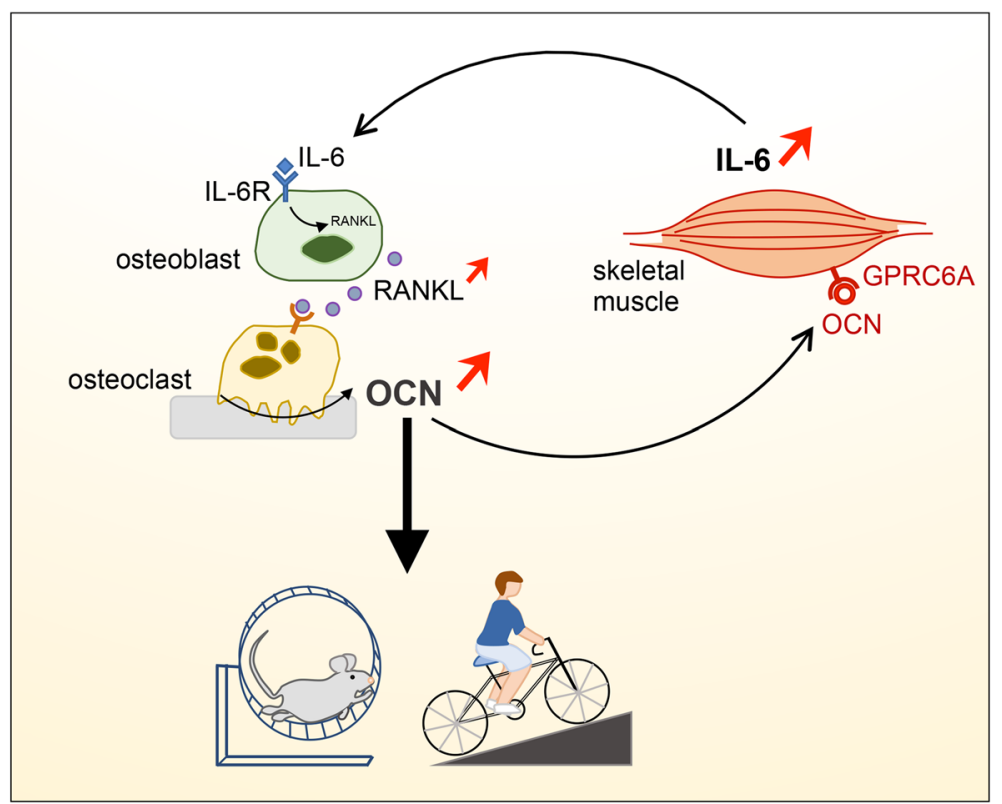

Figure 10. Schematic representation of how muscle-derived IL-6 increases exercise capacity in an osteocalcin-dependent manner. ously described ELISA (14). Total levels of osteocalcin and carboxylated osteocalcin were estimated using 2 different specific antibodies. Bioactive osteocalcin was determined by subtracting the carboxylated osteocalcin levels from total osteocalcin levels. CTX1 (Immunodiagnostic Systems [IDS], AC-06F1) and PiNP (IDS, AC-33F1) were also measured according to the manufacturer's instructions.

Circulating level of NEFAs, triglycerides, and glycerol were determined in serum using a colorimetric triglyceride assay kit (Biovision Inc., K622-100) and glycerol assay kit (Sigma-Aldrich, MAK117) according to the manufacturers' protocols.

Gene expression analysis by $q R T-P C R$. Muscles and other organs were snap-frozen in liquid nitrogen and kept at $-80^{\circ} \mathrm{C}$ until use. RNA was isolated using TRIzol (Invitrogen). RNA was first incubated with DNase I for $30 \mathrm{~min}$ utes at room temperature to remove any genomic DNA. DNase I-treated RNA was converted to cDNA by using M-MLV reverse transcriptase (Thermo Fisher Scientific, 28025013) and random hexamers (Thermo Fisher Scientific, N8080127). For gene expression, $1 \mathrm{mg}$ of RNA was reverse transcribed into cDNA. qPCR analyses were performed using PowerUp SYBER Green Master Mix (Applied Biosciences, Thermo Fisher Scientific). SYBR background. To minimize the confounding effect of a different genetic background, all experiments were performed using control littermates.

Mouse genotypes were determined by PCR. For osteocalcin treatment studies, $\mathrm{IlO}_{\mathrm{Hsa}}{ }^{-1-}$ and $\mathrm{IlOr}_{\mathrm{Osb}}{ }^{-1-}$ mice together with their controls were implanted with subcutaneous osmotic pumps (Alzet, model 1004) delivering osteocalcin $(120 \mathrm{ng} / \mathrm{h})$ for 28 days. After this period, mice were euthanized and muscles were dissected for analyses of muscle mass. Recombinant osteocalcin was purified as previously described (28).

Exercise. For exercise studies, all mice were trained to run on a treadmill for 4 days $(17 \mathrm{~min} /$ day, with increasing speed from 10 to 30 $\mathrm{cm} / \mathrm{s}$, and an electric shock at $0.4 \mathrm{~mA}$ to trigger running). Exercise tests were performed on mice fed ad libitum at 1-6 pm. On the test day, mice were acclimated to the treadmill for 5 minutes, followed by 10 minutes of running at a constant speed $(17 \mathrm{~cm} / \mathrm{s})$, followed by a gradual speed increase to $30 \mathrm{~cm} / \mathrm{s}$. Mice ran either until exhaustion to determine endurance capacity, or for 40 minutes. Mice were removed from the electric grid if the number of times a mouse fell off the grid during 1 minute reached 15 or more. For all biochemical and metabolic analyses, blood/tissues were collected and processed either at rest or at the end of a 40 -minute run $(30 \mathrm{~cm} / \mathrm{s})$. Injections (i.p.) of exogenous osteocalcin or IL-6 (Sigma-Aldrich, I9646) were performed immediately before exercise.

Biochemistry and molecular biology. Glucose and insulin tolerance tests were performed as described previously (29). Serum osteocalcin, IL-6, and glucagon levels were measured using ELISAs. Blood glucose level was measured using an Accu-Chek glucometer.

Circulatory IL-6levels were detected using Abcam System ELISA kits in the serum of human (Abcam, ab178013), monkey (Abcam, ab119549), rat (Abcam, ab100772), and mouse (Abcam, ab100712) according to the manufacturer's instructions. Blood was collected from the facial vein before and after exercise into tubes followed by centrifugation at $3000 \mathrm{~g}$ for 10 minutes at $4^{\circ} \mathrm{C}$ and serum aliquots were stored at $-80^{\circ} \mathrm{C}$ for further use. Mouse circulating osteocalcin levels were determined with a previGreen PCR conditions were 1 cycle of $50^{\circ} \mathrm{C}$ for 2 minutes, 1 cycle of $95^{\circ} \mathrm{C}$ for 10 minutes, and 40 cycles of $95^{\circ} \mathrm{C}$ for 15 seconds and $60^{\circ} \mathrm{C}$ for 60 seconds, using a model CFX96 Touch Real-Time PCR Detection System (Bio-Rad). Relative gene expression levels were calculated using the threshold cycle $\left(2^{-\Delta \Delta \mathrm{CT}}\right)$ method and normalized to Hprt.

Protein expression analysis by Western blot. Lysates were prepared from skeletal muscles using modified RIPA buffer $(150 \mathrm{mM} \mathrm{NaCl}, 1$ mM EDTA, $1 \%$ Triton X-100, $1 \%$ sodium deoxycholate, $0.1 \%$ SDS, and 20 mM Tris, $\mathrm{pH}$ 8.0) with protease inhibitors and $1 \mathrm{mM}$ sodium orthovanadate with protease inhibitor cocktail (Thermo Fisher Scientific, 862209) and homogenized before quantification by BCA assay (Thermo Fisher Scientific, 23225). After adjustment of the concentration $(100 \mu \mathrm{g} / 100 \mu \mathrm{L})$ in each of the samples, equal amounts of total protein $(20 \mu \mathrm{g})$ and SDS-Sample Buffer (Boston BioProducts, BP-110R) were boiled for 5 minutes at $98^{\circ} \mathrm{C}$, and recovered by spinning at $12,000 \mathrm{~g}$ for 5 minutes at $4^{\circ} \mathrm{C}$ before being loaded onto a $10 \%$ or $15 \%$ SDSPAGE gel. The gel was run for 80 minutes at $120 \mathrm{~V}$ in SDS-Running Buffer (Boston BioProducts, BP-177) before being transferred to a nitrocellulose membrane (Bio-Rad, 1620112) overnight at $25 \mathrm{~V}$. Blots were blocked in 5\% BSA (Applichem, A0830) in Tris-buffered saline (TBS) with $0.1 \%$ Tween for 1 hour before being incubated overnight in antibodies recognizing phospho HSL (Cell Signaling Technology, 4137S, 1:1000), HSL (Cell Signaling Technology, 4107, 1:1000), or GAPDH (Cell Signaling Technology, 5174S, 1:1000). Membranes were washed with TBST (137 mM NaCl, $2.7 \mathrm{mM} \mathrm{KCl,} 16.5 \mathrm{mM}$ Tris, pH 7.4, containing 0.1\% Tween 20) before being incubated with rabbit (Santa Cruz Biotechnology, SC-2004) secondary HRP-conjugated antibodies before being imaged using ECL substrate (Bio-Rad, 170-5061) on an ImageQuant LAS-4000 (GE Healthcare, 28-9607-59AB). Band intensities were quantified using Image J software (NIH).

In vivo glucose uptake. A modification of a previously described method (30) was used for in vivo glucose uptake. Before exercise, mice were injected with $10 \mu \mathrm{Ci}$ of ${ }^{3} \mathrm{H}-2-\mathrm{DG}$ in $100 \mu \mathrm{L}$ of $0.9 \% \mathrm{NaCl}$. 
Next, mice were placed on a treadmill and forced to run for 40 minutes at a constant speed $(30 \mathrm{~cm} / \mathrm{s})$. After exercise, blood and quadriceps muscles were collected to determine ${ }^{3} \mathrm{H}$ radioactivity. ${ }^{3} \mathrm{H}$ radioactivity in blood was similar in all mice, indicating similar systemic delivery of the tracer. White and red quadriceps muscles were homogenized in $1 \mathrm{~mL}$ of water immediately followed by boiling for $10 \mathrm{~min}$ utes. After that, homogenates were spun at max speed for 10 minutes. Fifty microliters of the supernatant was added to $450 \mu \mathrm{L}$ of water and counted in $5 \mathrm{~mL}$ of scintillation liquid. Nine hundred microliters were passed through an anion-exchange column (AG 1-X8 resin, Bio-Rad) to remove ${ }^{3} \mathrm{H}-2-\mathrm{DG}-6$-phosphate. The column was washed with $6 \mathrm{~mL}$ of water and $500 \mu \mathrm{L}$ of the eluted volume was counted in $5 \mathrm{~mL}$ of scintillation liquid. The difference between the total and eluted ${ }^{3} \mathrm{H}$ radioactivity represents ${ }^{3} \mathrm{H}-2-\mathrm{DG}-6$-phosphate accumulated in the tissue.

Recombinant osteocalcin. Mouse uncarboxylated osteocalcin was purified from E. coli BL21 transformed with pGEX2TK-mOCN, as described previously $(13,29)$. Briefly, GST-osteocalcin fusion protein was produced in BL21 pLyS transformed with pGEX2TK-mOCN after induction with IPTG. Cells were collected in lysis buffer $(1 \times$ PBS, 10 $\mathrm{mM}$ Tris $\mathrm{pH}$ 7.2, 2 mM EDTA, 1\% Triton X-100, and $1 \times$ protease and phosphatase inhibitor cocktail [Thermo Fisher Scientific, 78443]). Following 4 freeze/thaw cycles and sonication, lysates were cleared by centrifugation. The supernatant was incubated with glutathioneSepharose 4B (GE, 17075601) for 4 hours at $4^{\circ} \mathrm{C}$. Following 6 washes with washing buffer ( $1 \times$ PBS, $1 \%$ Triton X-100) and $1 \times$ PBS, osteocalcin was then cleaved from the GST moiety by using thrombin (GE, 27-0846-01). Four fractions were collected and each of them was incubated with benzamidine-Sepharose (GE, 17-5123-10) for $30 \mathrm{~min}$ utes at room temperature to remove thrombin.

Osteoblast/osteoclast coculture. Osteoblasts were obtained from 3- to 5-day-old mice using digestion medium containing $\alpha \mathrm{MEM}, 1.5$ $\mathrm{mg} / \mathrm{mL}$ collagenase $\mathrm{P}$, and $2.5 \%$ trypsin/EDTA. The cells were plated in 6- or 24-well plates in aMEM with FBS (not heat inactivated) and cultured for 48 to 60 hours until confluent. For adenovirus infection, 50,000 to 75,000 cells/well (24-well plate) were plated and infection was performed the next day. Cultures were kept in the $\alpha \mathrm{MEM}$ with $10 \%$ FBS until they reached confluence.

When osteoblast cultures were confluent we prepared the bone marrow cells from tibias and femurs of 4- to 10-week-old mice. Extremities of each bone were cut with scissors before flushing the bone marrow into a $15-\mathrm{mL}$ Falcon tube using a 10-mL syringe connected to a $25-G 5 / 8$-in. needle. The cell suspension was homogenized by gentle pipetting and washed twice with $\alpha \mathrm{MEM}$ and resuspended in 5 $\mathrm{mL}$ media. The cells were counted with a hemacytometer and plated in $\alpha \mathrm{MEM}$ for the desired density of $0.3 \times 10^{6}$ to $3 \times 10^{6}$ cells/well. The medium was supplemented with serum from Il6-null mice $(10 \%)$ and supplemented with PGE2 $\left(1 \times 10^{-6} \mathrm{M}\right)$ and vitamin D3 $\left(1 \times 10^{-6} \mathrm{M}\right)$ to promote RankL and M-CSF expression by osteoblasts, leading to osteoclast formation. IL-6 (10 ng/mL) and sIL-6R (100 ng/mL) were added $(21,31)$. Half of the medium was changed every 2 days with extreme care not to aspirate the myeloid progenitor cells sitting on the osteoblasts. After coculturing for the next 5 to 8 days, the osteoblast layer was removed by washing 2 times with $1 \times$ PBS and then adding $500 \mu \mathrm{L}$ of prewarmed digestion medium ( $\alpha \mathrm{MEM}+0.1 \%$ collagenase $+0.2 \%$ Dispase) at $37^{\circ} \mathrm{C}$ for 5 to 15 minutes by carefully monitoring if osteoblasts were detaching and removing the osteoblast layer completely. Once osteoblasts were detached, osteoclasts were washed again twice with $1 \times$ PBS and fixed with paraformaldehyde (PFA) or formalin for TRAP assay or lysed in TRIzol for RNA expression analysis.

Adenoviral transduction of primary osteoblasts and bone marrowderived osteoclast precursor cells. Adenoviral vector with the cytomegalovirus (CMV) promoter (Ad5CMVEmpty) that expresses no protein and adenoviral vector with CMV driving the expression of Cre recombinase (Ad5CMVCRE) with a titer of $1 \times 10^{10} \mathrm{PFU} / \mathrm{mL}$ were purchased from the University of Iowa Viral Vector Core. Mouse osteoblasts and bone marrow-derived osteoclast precursor cells were transduced the day after they were plated into multiwell tissue culture plates with cell culture grade poly-L-lysine (PLL) (Sigma-Aldrich, $0.5 \mu \mathrm{L} / \mathrm{mL}$ ). Serumfree $\alpha \mathrm{MEM}$ was prepared at $50 \%$ of the typical well cell culture volume ( $0.5 \mathrm{~mL} /$ well of a 12-well plate). PLL was added just before addition of virus (MOI 200) and was allowed to incubate for 10 minutes at room temperature. The virus-containing medium was used to replace the culture medium and cells were returned to the incubator. After a transduction period of 12 hours, the viral medium was discarded and replaced with fresh complete medium (32).

Osteocalcin and IL-6 injections. For all biochemical and metabolic analyses, blood and tissues were collected and processed either at rest or at the end of a 50-minute run $(30 \mathrm{~cm} / \mathrm{s})$. Injections (i.p.) of osteocalcin or IL-6 (MilliporeSigma) were performed immediately before exercise. To neutralize IL-6, $500 \mathrm{mg}$ of a neutralizing antibody (R\&D Systems, MAB206) (33) was administered i.p. 1 hour before exercise.

Immunohistochemistry. A whole-body transcardial perfusion was done with saline followed by fresh $4 \%$ PFA. Skeletal muscle was dissected with as little force tension manipulation as possible to maintain the overall fusiform structure to determine the axis for critical transverse embedding. The dissected muscle sample was placed in a dry weight dish for 2 to 3 minutes to allow for recovery from manipulation and then immersed in 20 volumes minimum of fresh $4 \%$ PFA for 16 to 24 hours for fixation at room temperature with agitation sufficient to move the tissue. Samples were then transfer to $1 \times$ PBS in sealed vials without any air bubbles and processed for paraffin embedding.

Muscle sections (5- $\mu \mathrm{m}$-thick) were prepared, deparaffinized with xylene, and rehydrated with a graded series of ethanol (absolute, 95\%, $90 \%, 80 \%$, and $70 \%$ in water). Slides were incubated in ice-cold permeabilization solution $(0.1 \%$ Triton $\mathrm{X}-100$ and $0.1 \%$ sodium citrate in water) and blocked in PBS containing 10\% BSA and 10\% normal goat serum. Sections were stained with NOQ7 monoclonal antibody for myosin heavy chain isoform I (MHC I) slow twitch (Sigma-Aldrich, M4276) and MY32 monoclonal antibody for MHC II fast twitch (SigmaAldrich, M8421), followed by secondary antibody anti-mouse IgGperoxidase and staining (Sigma-Aldrich, A8924). Images were captured with a Nikon A1 confocal microscope.

Bone histology and histomorphometry. Six-month-old Il6rl/fl and $I l 6 r_{O s b}{ }^{-1-}$ mice were euthanized 1 week after the last exercise. Lumbar vertebrae and tibiae from 6-month-old $I l 6 r^{f / / f l}$ and $I l 6 r_{O b s}{ }^{-1-}$ mice were dissected, fixed in $10 \%$ buffered formalin for 24 hours, and dehydrated through a series of increasing ethanol concentrations. Samples were then embedded in methylmethacrylate (MMA) according to standard protocols. Four- and 8- $\mu \mathrm{m}$-thick serial sections were cut and von Kossa, TRAP, and toluidine blue staining were used to measure trabecular bone volume versus tissue volume (BV/TV), osteoclast number, and osteoblast number, respectively. Bone histomorphometry analysis was performed on at least 30 fields at $\times 40$ magnification as described previously (34) using the Osteomeasure Analysis system (Osteometrics). 
Statistics. All values are depicted as mean \pm SEM. Statistical parameters including the exact value of $n$, post hoc test, and statistical significance are reported in every figure and figure legends. Data were determined to be statistically significant when $P<0.05$ by Student's $t$ test or 1-way or 2-way ANOVA. Data were analyzed using GraphPad Prism 7.

Study approval. All human subjects signed a written informed consent before participating and were free to withdraw from the study at any time. The study was conducted in accordance with the guidelines for Good Clinical Practice and the Declaration of Helsinki. The study was approved by the Region Ethical Committee, Copenhagen, Denmark (H-16018062) with the registration at Clinicaltrials.gov as NCT02901496. The results have been reported in accordance with the CONSORT guidelines. All primate procedures were approved by the Institutional Animal Ethics Committee for the Care and Use of Primates in Research at the National Institute of Immunology, New Delhi, India (IAEC/2017/157). All experiments involving mice were approved by the Institutional Animal Care and Use Committee of the Columbia University Medical Center.

\section{Author contributions}

SC and GK conceived of the study and designed experiments. SC, LS, BP, PS, JMB, VKY, and PM performed experiments. HH contributed sera from patients. JH and JB provided floxed mice. SC and GK analyzed data and wrote the manuscript.

\section{Acknowledgments}

We thank Gerald Shulman (Yale University), Patricia Ducy (Columbia University), and Mathieu Ferron (Montreal Clinical Research Institute-IRCM) for advice, reagents, and reading the manuscript. This work was supported by 5R01DK104727-05 (NIDDK) (to GK), 1R01AR073180-01A1 (NIAMS) (to GK), Canadian Institute of Health and Research (CIHR) Fellowship (201511MFE-359182-181537) (to SC), Ramalingaswamy Fellowship (BT/HRD/35/02/2006), Department of Biotechnology, India (to VKY), Ministerio de Economía y Competitividad y Fondo Europeo de Desarrollo Regional SAF201456546-R and RTI2018-101105-B-I00 (to JH), TrygFonden (grants ID 101390 and ID 20045) (to HE).
1. Neufer PD, et al. Understanding the cellular and molecular mechanisms of physical activity-induced health benefits. Cell Metab. 2015;22(1):4-11.

2. Zierath JR, Wallberg-Henriksson H. Looking ahead perspective: where will the future of exercise biology take us? Cell Metab. 2015;22(1):25-30.

3. Pedersen BK, Febbraio MA. Muscles, exercise and obesity: skeletal muscle as a secretory organ. Nat Rev Endocrinol. 2012;8(8):457-65.

4. Ostrowski K, Rohde T, Zacho M, Asp S, Pedersen BK. Evidence that interleukin- 6 is produced in human skeletal muscle during prolonged running. J Physiol (Lond). 1998;508(pt 3):949-953.

5. Steensberg A, van Hall G, Osada T, Sacchetti M, Saltin B, Klarlund Pedersen B. Production of interleukin- 6 in contracting human skeletal muscles can account for the exercise-induced increase in plasma interleukin-6. JPhysiol (Lond). 2000;529(pt 1):237-242.

6. Pedersen BK, et al. Searching for the exercise factor: is IL-6 a candidate? JMuscle Res Cell Motil. 2003;24(2-3):113-119.

7. Lang Lehrskov L, et al. Interleukin-6 delays gastric emptying in humans with direct effects on glycemic control. Cell Metab. 2018;27(6):1201-1211.e3.

8. Wedell-Neergaard AS, et al. Exercise-induced changes in visceral adipose tissue mass are regulated by IL- 6 signaling: a randomized controlled trial. Cell Metab. 2019;29(4):844-855.e3.

9. Febbraio MA, Hiscock N, Sacchetti M, Fischer CP, Pedersen BK. Interleukin- 6 is a novel factor mediating glucose homeostasis during skeletal muscle contraction. Diabetes. 2004;53(7):1643-1648.

10. van Hall G, et al. Interleukin-6 stimulates lipolysis and fat oxidation in humans. J Clin Endocrinol Metab. 2003;88(7):3005-3010.

11. Catoire M, Kersten S. The search for exercise factors in humans. FASEB J. 2015;29(5):1615-1628.

12. Hawley JA, Hargreaves M, Joyner MJ, Zierath JR. Integrative biology of exercise. Cell. 2014;159(4):738-749.
13. Mera $\mathrm{P}$, et al. Osteocalcin signaling in myofibers is necessary and sufficient for optimum adaptation to exercise. Cell Metab. 2016;23(6):1078-1092.

14. Ferron M, et al. Insulin signaling in osteoblasts integrates bone remodeling and energy metabolism. Cell. 2010;142(2):296-308.

15. Johnson RW, et al. The primary function of gp130 signaling in osteoblasts is to maintain bone formation and strength, rather than promote osteoclast formation. JBone Miner Res. 2014;29(6):1492-1505

16. McCarthy JJ, Srikuea R, Kirby TJ, Peterson CA, Esser KA. Correction: Inducible Cre transgenic mouse strain for skeletal muscle-specific gene targeting. Skelet Muscle. 2012;2(1):22.

17. Miniou P, Tiziano D, Frugier T, Roblot N, Le Meur M, Melki J. Gene targeting restricted to mouse striated muscle lineage. Nucleic Acids Res. 1999;27(19):e27.

18. von Maltzahn J, Jones AE, Parks RJ, Rudnicki MA. $\mathrm{Pax} 7$ is critical for the normal function of satellite cells in adult skeletal muscle. Proc Natl Acad Sci U S A. 2013;110(41):16474-16479.

19. Suda T, Takahashi N, Martin TJ. Modulation of osteoclast differentiation. Endocr Rev. 1992;13(1):66-80.

20. Mihara M, Hashizume M, Yoshida H, Suzuki M, Shiina M. IL-6/IL-6 receptor system and its role in physiological and pathological conditions. Clin Sci. 2012;122(4):143-159.

21. McGregor NE, et al. IL-6 exhibits both cis- and trans-signaling in osteocytes and osteoblasts, but only trans-signaling promotes bone formation and osteoclastogenesis. J Biol Chem. 2019;294(19):7850-7863.

22. Zhang M, et al. Osteoblast-specific knockout of the insulin-like growth factor (IGF) receptor gene reveals an essential role of IGF signaling in bone matrix mineralization. J Biol Chem. 2002;277(46):44005-44012.

23. Wunderlich FT, et al. Interleukin- 6 signaling in liver-parenchymal cells suppresses hepatic inflammation and improves systemic insulin action. Cell Metab. 2010;12(3):237-249.

24. Perry RJ, et al. Hepatic acetyl CoA links adipose tissue inflammation to hepatic insulin resistance and type 2 diabetes. Cell. 2015;160(4):745-758.

25. Stahl A, Gimeno RE, Tartaglia LA, Lodish HF. Fatty acid transport proteins: a current view of a growing family. Trends Endocrinol Metab. 2001;12(6):266-273.

26. Mera P, Laue K, Wei J, Berger JM, Karsenty G. Corrigendum to "Osteocalcin is necessary and sufficient to maintain muscle mass in older mice" [Mol Metabol 5 (2017) 1042-1047]. Mol Metab. 2017;6(8):941.

27. Berger JM, et al. Mediation of the acute stress response by the skeleton. Cell Metab. 2019;30(5):890-902.e8.

28. Oury F, et al. Maternal and offspring pools of osteocalcin influence brain development and functions. Cell. 2013;155(1):228-241.

29. Lee NK, et al. Endocrine regulation of energy metabolism by the skeleton. Cell. 2007;130(3):456-469.

30. Howlett KF, Andrikopoulos S, Proietto J, Hargreaves M. Exercise-induced muscle glucose uptake in mice with graded, muscle-specific GLUT-4 deletion. Physiol Rep. 2013;1(3):e00065.

31. Tamura T, et al. Soluble interleukin-6 receptor triggers osteoclast formation by interleukin 6. Proc Natl Acad Sci U S A. 1993;90(24):11924-11928.

32. Buo AM, Williams MS, Kerr JP, Stains JP. A costeffective method to enhance adenoviral transduction of primary murine osteoblasts and bone marrow stromal cells. Bone Res. 2016;4:16021.

33. Pedersen L, et al. Voluntary running suppresses tumor growth through epinephrine- and IL-6dependent NK cell mobilization and redistribution. Cell Metab. 2016;23(3):554-562.

34. Yadav VK, et al. Lrp5 controls bone formation by inhibiting serotonin synthesis in the duodenum. Cell. 2008;135(5):825-837. 